\title{
Mode transition of electromagnetic wave in slab waveguide subjected to in-plane elastic
}

\section{wave}

\author{
Chau-Shioung Yeh ${ }^{\mathrm{a}, *}$ and Chun-Bo Lin ${ }^{\mathrm{b}}$ \\ anstitute of Applied Mechanics, National Taiwan University, Taipei, Taiwan 10764, R.O.C. \\ Tel: +8862 23630979; Fax: +8862 29322714; E-mail: csyeh@spring.iam.ntu.edu.tw \\ ${ }^{\mathrm{b}}$ Department of Mechanical Engineering, Nan Kai Junior College, Nantau, Taiwan 542, R.O.C. \\ E-mail: cblin@bear.nkjc.edu.tw
}

Received 17 August 1999

Revised 15 February 2000

\begin{abstract}
In this paper, on the basis of electromagnetic fields in statistical formulation and the material description for solid medium, the governing equations are decomposed into different states with the necessary approximations. The relevant equations with boundary equations are applied to investigate the phase change of electromagnetic wave and the mode transition of guided electromagnetic wave in a slab waveguide, which is caused by the mechanical disturbance such as strain or elastic wave. The conditions of the mode transition for TE mode are determined and the physical meaning of the results is interpreted.
\end{abstract}

\section{Introduction}

The mechanical disturbance (strain, etc.) will influence the propagation of electromagnetic wave in the optical fiber. Bucaro et al. [1] have used Mach-Zehnder arrangement to measure the relative phase change between a active fiber and a reference fiber and calculate it by applying photoelastic theory. Although the real strain field in cylindrical fiber has been used in the calculation basing on photoelastic theory [2,3], the major deviation between the real physical situation and the calculation numerical results presents in the ultrasonic range of elastic wave. The main reason is that the boundary conditions of electromagnetic field can not be introduced in the photoelastic theory.

A singular surface may exist at the position of discontinuity of the electromagnetic or mechanical field. The order of singular surface depends on the first occurance of jump for the sequential derivative of the position vector. In general, the interface between different electromagnetic materials can be regarded as the singular surface of order two. The jump condition at such a surface can be regarded as boundary conditions in the problems which we will deal with. The earlier investigators have formulated the boundary conditions of the electromagnetic field in spatial description [4-10] or in material description [8, $11-14]$, some authors $[5,11,12]$ have listed the boundary conditions of perturbed fields as well as the original fields.

\footnotetext{
${ }^{*}$ Corresponding author.
} 
In our previous papers, we have investigated the relative phase change of the electromagnetic wave disturbed by static strain [14] or in-plane elastic wave [15] in an infinite space and shown the equivalence of results on the basis of the deformable electromagnetic theory and the photoelastic theory. Nevertheless, since the photoelastic theory lacks a formal mechanical basis, which is not adequate to be used to treat the finite dimension problem. Therefore, the boundary conditions of electromagnetic and mechanical fields on dielectric waveguide derived in the following paragraph can be introduced in the deformable electrodynamic theory only. In this paper, we will study the change of guided electromagnetic wave in slab waveguide which is surrounded by vacuum. In order to keep the consistency in application, the jump conditions of statistical formulation in material description for the guided electromagnetic wave in rigid body state and the perturbed electromagnetic wave are derived. Thereafter, the mode transition of the original guided electromagnetic wave can be determined.

Since the basic definition of electromagnetic fields in statistical formulation is the most popular one in various electromagnetic models, we adopt such a formulation in this paper. Some fundamental governing equations and approximations need to be introduced in Section 2. In Section 3, the jump conditions for the rigid body state and the perturbed electromagnetic fields are formulated in the dielectric side and the vacuum side, respectively. The electromagnetic fields in rigid body state and perturbed state will be determined in Section 4. Besides, the relative phase change and the mode transition of the original guided electromagnetic wave, which is a new result, can also be obtained. Some investigations for the condition of mode transition are shown in Section 5.

\section{Governing equations}

Although Hutter and van de Ven [8] have shown that the equivalence of the various electrodynamic models under non-relativistic approximation, the statistical formulation is adopted here because of its more sophisticate physical foundation [16] and widespread in application. The differential form of governing equations in material configuration can be rewritten from Ref.8 with proper modification as follows.

A. Electromagnetic field equations [10]

$$
\begin{aligned}
& \bar{\nabla} \times \mathcal{E}+\frac{d}{d t} \mathcal{B}=0, \quad \bar{\nabla} \cdot \mathcal{B}=0, \quad \bar{\nabla} \cdot \mathcal{D}=\mathcal{Q} \\
& \bar{\nabla} \times \mathcal{H}=\mathcal{J}+\frac{d}{d t} \mathcal{D}, \quad \bar{\nabla} \cdot \mathcal{J}+\frac{d}{d t} \mathcal{Q}=0
\end{aligned}
$$

where del operator $\bar{\nabla}$ denotes the differentiation with respect to the material coordinates $\mathbf{X} . \mathcal{B}, \mathcal{H}, \mathcal{D}, \mathcal{E}$, $\mathcal{J}, \mathcal{Q}$ denote magnetic flux, effective magnetic field, electric displacement, effective electric field,electric current and electric charge, respectively in the material state. The corresponding transformation for those fields $\mathbf{B}, \mathbf{H}, \mathbf{D}, \mathbf{E}, \mathbf{J}, \mathrm{Q}$ in spatial frame to material one can be listed as

$$
\begin{aligned}
& \mathcal{E}=\mathbf{E}^{e} \cdot \mathbf{F}, \quad \mathcal{H}=\mathbf{H}^{e} \cdot \mathbf{F}, \quad \mathcal{M}=\mathbf{M} \cdot \mathbf{F}, \quad \mathcal{B}=\mathbf{B} \cdot J \mathbf{F}^{-T} \\
& \mathcal{D}=\mathbf{D} \cdot J \mathbf{F}^{-T}, \quad \mathcal{P}=\mathbf{P} \cdot J \mathbf{F}^{-T}, \quad \mathcal{J}=\mathbf{J} \cdot J \mathbf{F}^{-T}, \quad \mathcal{Q}=J Q
\end{aligned}
$$

where

$$
\mathbf{E}^{\mathbf{e}}=\mathbf{E}+\dot{\mathbf{x}} \times \mathbf{B}, \quad \mathbf{D}=\varepsilon_{0} \mathbf{E}+\mathbf{P}
$$




$$
\begin{aligned}
& \mathbf{H}^{\mathbf{e}}=\mathbf{H}-\dot{\mathbf{x}} \times \mathbf{D}, \quad \mathbf{H}=\frac{1}{\mu_{0}} \mathbf{B}-\mathbf{M} \\
& \mathbf{F}^{-T}=\left(\mathbf{F}^{-1}\right)^{T}
\end{aligned}
$$

The deformation gradient $\mathbf{F}$ connects the differential position vectors before and after the deformation (i.e. $\mathrm{d} \mathbf{x}=\mathrm{d} \mathbf{F} \cdot \mathbf{X}$ ). The relations among $\mathcal{H}, \mathcal{B}, \mathcal{D}, \mathcal{E}$ can be written as

$$
\begin{aligned}
\mathcal{H} & =\frac{1}{\mu_{0} J}(\mathbf{C} \cdot \mathcal{B})-\left(\mathbf{F}^{-1} \cdot \dot{\mathbf{x}}\right) \times \mathcal{D}-\mathcal{M} \\
\mathcal{D} & =\varepsilon_{0}\left[J\left(\mathbf{C}^{-1} \cdot \mathcal{E}\right)-\frac{1}{J}(\dot{\mathbf{x}} \cdot \mathbf{F}) \times(\mathbf{C} \cdot \mathcal{B})\right]+\mathcal{P} \simeq \varepsilon_{0} J\left(\mathbf{C}^{-1} \cdot \mathcal{E}\right)+\mathcal{P}
\end{aligned}
$$

where $\mathbf{C}\left(=\mathbf{F}^{T} \cdot \mathbf{F}\right)$ denotes the Green deformation tensor. The semi-relativistic approximation [8] which can be used to drop those terms with the order of magnitude $|\dot{\mathbf{x}}|^{2} / \mathrm{c}^{2}$ (c : speed of light in body) is adopted in the derivation of the last approximation in (4b).

B. Balance laws of mechanics

$$
\begin{aligned}
& \dot{\rho}^{R}=0, \quad \rho^{R} \ddot{\mathbf{x}}=\bar{\nabla} \cdot \mathbf{T}^{T}+\rho^{R} \mathbf{f}, \quad \mathbf{F}^{T} \star \mathbf{T}+\rho^{R} \mathbf{L}=0 \\
& \rho^{R} \dot{U}=\dot{\mathbf{F}}^{T}: \mathbf{T}-\bar{\nabla} q+\rho^{R} r, \quad \rho^{R} \dot{\eta}+\bar{\nabla} \cdot \varphi-\rho^{R} s \geqslant 0
\end{aligned}
$$

where

$$
\begin{aligned}
\rho^{R} \mathbf{f}= & \left\{(\mathcal{Q}-\bar{\nabla} \cdot \mathcal{P}) \mathcal{E}+(\mathcal{J}+\dot{\mathcal{P}}) \times \mathcal{B}+\left[\bar{\nabla} \mathcal{M}^{\mathrm{L}}-\left(\bar{\nabla} \mathcal{M}^{\mathrm{L}}\right)^{T}\right] \cdot \mathcal{B}+\bar{\nabla} \cdot\left(\mathcal{E} \otimes \mathcal{P}-\mathcal{M}^{\mathrm{L}} \otimes \mathcal{B}\right.\right. \\
& \left.\left.+\mathcal{M}^{\mathrm{L}} \cdot \mathcal{B} \mathbf{I}\right)\right\} \cdot \mathbf{F}^{-1}, \quad \rho^{R} \mathbf{L}=\left(\mathbf{F} \otimes \mathbf{F}^{-1}-\mathbf{F}^{T} \otimes \mathbf{F}^{-T}\right):\left(\mathcal{P} \otimes \mathcal{E}-\mathcal{B} \otimes \mathcal{M}^{\mathrm{L}}\right) \\
\rho^{R} r= & (\mathcal{J}+\dot{\mathcal{P}}) \cdot \mathcal{E}-\mathcal{M}^{\mathrm{L}} \cdot \mathcal{B}+\dot{\mathbf{F}} \cdot\left[\left(\mathcal{P} \otimes \mathcal{E}-\mathcal{B} \otimes \mathcal{M}^{\mathrm{L}}\right)+\left(\mathcal{M}^{\mathrm{L}} \cdot \mathcal{B}\right) \mathbf{I}\right] \cdot \mathbf{F}^{-1}
\end{aligned}
$$

The notations $\rho^{R}, q$ and $\varphi$ denote material density, heat flux and entropy flux in material state. The quantities used are the first Piola-Kirchhoff stress tensor $\mathbf{T}$, body force $\mathbf{f}$, body couple $\mathbf{L}$, internal energy $U$, internal energy supply $r$, entropy $\eta$, entropy supply $s$ and second order unit tensor I. Symbols $\otimes$ and : stand for tensor product and contracted product, respectively. The notation $\star$ denotes that vector product is taken for the first inices of both second tensor and the scalar product is conducted for the second indices. The convenient notation $\mathcal{M}^{\mathrm{L}}$ which has been used in the compact expression (6) can be expressed as

$$
\begin{aligned}
\mathcal{M}^{\mathrm{L}} & =(\mathbf{M}+\dot{\mathbf{x}} \times \mathbf{P}) \cdot \mathbf{F}=-\mathcal{H}+\frac{1}{\mu_{0} J} \mathbf{C}^{\mathrm{L}} \cdot \mathcal{B}-\frac{\varepsilon_{0}}{J} \mathbf{C} \cdot[(\dot{\mathbf{x}} \cdot \mathbf{F}) \times \mathcal{E}] \\
& =\mathcal{M}-\left(\mathbf{F}^{-1} \cdot \dot{\mathbf{x}}\right) \times \mathcal{D}-\frac{\varepsilon_{0}}{J} \mathbf{C} \cdot[(\dot{\mathbf{x}} \cdot \mathbf{F}) \times \mathcal{E}]
\end{aligned}
$$

with

$$
\mathbf{C}^{\mathrm{L}}=\left(1-\frac{|\dot{\mathbf{x}}|^{2}}{\mathrm{c}^{2}}\right) \mathbf{C}+\frac{1}{\mathrm{c}^{2}}(\dot{\mathbf{x}} \mathbf{F}) \otimes(\dot{\mathbf{x}} \mathbf{F}) \simeq \mathbf{C}
$$

C. Constitutive equations

On the basis of representation theorem, the constitutive equation of dielectric can be represented by a scalar function as [14]

$$
\gamma=\frac{1}{2 \rho^{w}}\left\{C_{I}+C_{I I}\left(\operatorname{tr} \mathrm{E}^{w}\right)+C_{I I I}\left(\operatorname{tr} \mathrm{E}^{w}\right)^{2}+C_{I V}\left[\operatorname{tr}\left(\mathrm{E}^{w} \cdot \mathrm{E}^{w}\right)\right]+C_{V}\left(\mathcal{E}^{w} \cdot \mathrm{E}^{w} \cdot \mathcal{E}^{w}\right)\right\}
$$


where

$$
\rho^{w}=\rho J^{w}, \quad \mathcal{P}^{w}=\mathbf{P} \cdot J^{w}\left(\mathbf{F}^{w}\right)^{-T}, \quad \mathrm{E}^{w}=\frac{1}{2}\left(\mathbf{C}^{w}-\mathbf{I}\right)=\frac{1}{2}\left[\left(\mathbf{F}^{w}\right)^{T} \cdot \mathbf{F}^{w}-\mathbf{I}\right]
$$

The quantities with superscript $w$ are the corresponding one in natural state. Thereafter, by basing on the definition of field quantity $[8,14]$,

$$
\mathcal{P}^{w}=-\rho^{w} \frac{\partial \gamma}{\partial \mathcal{E}^{w}}, \quad \mathcal{M}^{w}=-\rho^{w} \frac{\partial \gamma}{\partial \mathcal{B}^{w}}, \quad \mathbf{T}^{w}=\rho^{w} \frac{\partial \gamma}{\partial \mathrm{E}^{w}} \cdot\left(\mathbf{F}^{w}\right)^{T}
$$

and the transformations related to predeformation as,

$$
\mathcal{E}^{w}=\mathcal{E} \cdot \mathbf{F}^{p}, \quad \mathcal{B}^{w}=\mathcal{B} \cdot J^{p}\left(\mathbf{F}^{p}\right)^{-T}, \quad \mathcal{Q}^{w}=J^{p} \mathcal{Q}, \quad J^{p}=\operatorname{det} \mathbf{F}^{p}, \quad \text { etc. }
$$

the constitutive equations in which the predeformation is included implicitly can be obtained as follows,

$$
\begin{aligned}
& \mathcal{P}=\left[\mathrm{P}^{I}+\mathrm{P}^{I I}:\left(\mathrm{E}+\mathrm{E}^{p}\right)\right] \cdot \mathcal{E}, \quad \mathcal{M}=\mathcal{Q}=\mathcal{J}=0 \\
& \mathbf{T}=\left[\mathcal{C}:\left(\mathrm{E}+\mathrm{E}^{p}\right)+(\mathcal{E} \cdot \mathcal{E}) \mathrm{T}^{I}+\mathrm{P}^{I I I}:(\mathcal{E} \otimes \mathcal{E})\right]
\end{aligned}
$$

where

$$
\begin{aligned}
& \mathrm{P}^{I}=\frac{1}{J^{p}} P_{I} \mathrm{~B}^{p}, \quad \mathrm{P}^{I I}=\frac{1}{J^{p}}\left(P_{I I} \mathrm{~B}^{p} \mathrm{~B}^{p}+P_{I I I} \mathrm{~B}^{p} \mathrm{~B}^{p}\right), \quad \mathrm{P}^{I I I}=-\frac{1}{J^{p}} P_{I I I}\left(\mathrm{~B}^{p} \mathrm{~B}^{p}\right) \\
& \mathcal{C}=\frac{1}{J^{p}}\left(\lambda \mathrm{B}^{p} \mathrm{~B}^{p}+2 \mu \mathrm{B}^{p} \mathrm{~B}^{p}\right), \quad \mathrm{T}^{I}=-\frac{1}{2 J^{p}} P_{I I} \mathrm{~B}^{p}
\end{aligned}
$$

with

$$
\begin{aligned}
& P_{I}=-\left[\frac{\partial C_{I}}{\partial \mathcal{E}^{w^{2}}}+\frac{\partial C_{V}}{\partial \mathcal{E}^{2}}\left(\mathcal{E}^{w} \cdot \mathrm{E}^{w} \cdot \mathcal{E}^{w}\right)\right], \quad P_{I I}=-\frac{\partial C_{I I}}{\partial \mathcal{E}^{2}}, \quad P_{I I I}=-C_{V} \\
& \lambda=C_{I I I}, \quad \mu=\frac{1}{2} C_{I V}, \quad T_{I}=\frac{1}{2} C_{I I}=-\frac{1}{2} \int P_{I I} d \mathcal{E}^{w 2}=-\frac{1}{2} P_{I I} \mathcal{E}^{w 2} \\
& \mathrm{E}^{p}=\frac{1}{2}\left[\mathbf{I}-\left(\mathrm{B}^{p}\right)^{-1}\right], \quad \mathrm{B}^{p}=\mathbf{F}^{p} \cdot\left(\mathbf{F}^{p}\right)^{T}, \quad\left(\mathrm{~B}^{p} \mathrm{~B}^{p}\right): \mathrm{E}=\mathrm{B}^{p} \cdot \mathrm{E} \cdot \mathrm{B}^{p}
\end{aligned}
$$

Here $P_{I}$ can be regarded as electric susceptibility, $P_{I I}$ and $P_{I I I}$ are electrostriction constants, $\lambda$ and $\mu$ are Lamé's constants, $T_{I}$ shows the effect of interaction between electric field and matter. Note that the quantities with superscript $p$ are related to the predeformation.

D. Linearization and rigid body state approximation

Since the equations listed above are highly nonlinear and hard to be solved, the linearization process is needed to deal with the real problem undertaken. The sequence of the applied load and field process mentioned in Ref.14 is also valid here. The relationship between the position vectors of various states can be written as

$$
\mathrm{X}_{\mathrm{A}}=\delta_{\mathrm{A} \Gamma} \Xi_{\Gamma}+\mathrm{U}_{\mathrm{A}}, \quad \xi_{\alpha}=\delta_{\alpha \mathrm{A}}\left(\mathrm{X}_{\mathrm{A}}+\overline{\mathrm{U}}_{\mathrm{A}}\right), \quad \mathrm{x}_{i}=\delta_{i \alpha}\left(\xi_{\alpha}+\delta_{\alpha \mathrm{A}} \mathrm{u}_{\mathrm{A}}\right)
$$

where position vectors are $\Xi_{\Gamma}, \mathrm{X}_{\mathrm{A}}, \xi_{\alpha}, \mathrm{x}_{\mathrm{i}}$ in natural, initial, intermediate and final states, respectively. $\mathrm{U}_{\mathrm{A}}, \overline{\mathrm{U}}_{\mathrm{A}}$ and $\mathrm{u}_{\mathrm{A}}$ denote the displacements which are caused by predeformation, originally applied electromagnetic fields, and mechanical disturbance, respectively. Note that the initial state is used as 
reference state in this paper. The symbols $\delta_{A \Gamma}, \delta_{\alpha A}$ and $\delta_{i A}$ denote Kronecker delta and are the shift factors for different states. The terms related to predeformation are all contained in the coefficient of constitutive equation as shown above. Therefore, $\mathrm{U}_{\mathrm{A}}$ will not appear explicitly herein. Furthermore, by the order estimation $\left|\overline{\mathrm{U}}_{\mathrm{A}, \mathrm{B}}\right| \ll\left|\mathrm{u}_{\mathrm{A}, \mathrm{B}}\right| \ll 1$ made in the appendix of Ref.14, those terms (such as $\overline{\mathrm{e}}_{\mathrm{A}}, \overline{\mathrm{b}}_{\mathrm{A}}$, etc.) with the same order of magnitude of $\bar{U}_{A, B}$ are negligible. Such an approximation will lead to a rigid body state which experiences the originally applied electromagnetic field rigidly then take the same position vector as initial state. Therefore, we can obtain the following approximation:

$$
\begin{aligned}
& \mathrm{F}_{\mathrm{iA}}=\mathrm{x}_{\mathrm{i}, \mathrm{A}}=\delta_{i \alpha}\left(\overline{\mathrm{F}}_{\alpha \mathrm{A}}+\delta_{\alpha \mathrm{B}} \mathrm{u}_{\mathrm{B}, \mathrm{A}}\right) \simeq \delta_{i \alpha}\left(\delta_{\alpha \mathrm{A}}+\delta_{\alpha \mathrm{C}} \mathrm{U}_{\mathrm{C}, \mathrm{A}}^{-}+\delta_{\alpha \mathrm{B}} \mathrm{u}_{\mathrm{B}, \mathrm{A}}\right) \simeq \delta_{i \alpha}\left(\delta_{\alpha \mathrm{A}}+\delta_{\alpha \mathrm{B}} \mathrm{u}_{\mathrm{B}, \mathrm{A}}\right) \\
& \mathrm{F}_{\mathrm{Ai}}^{-1} \simeq \delta_{\mathrm{Ai}}-\mathrm{u}_{\mathrm{A}, \mathrm{B}} \delta_{\mathrm{Bi}}, \quad J \simeq 1+\mathrm{u}_{\mathrm{B}, \mathrm{B}}, \quad \mathrm{E}_{\mathrm{AB}} \simeq \frac{1}{2} \mathrm{u}_{(\mathrm{A}, \mathrm{B})} \\
& \mathrm{C}_{\mathrm{AB}} \simeq \delta_{\mathrm{AB}}+\mathrm{u}_{(\mathrm{A}, \mathrm{B})}, \quad \mathrm{C}_{\mathrm{AB}}^{-1} \simeq \delta_{A B}-\mathrm{u}_{(\mathrm{A}, \mathrm{B})} \\
& \mathcal{E}_{\mathrm{A}}=\overline{\mathcal{E}}_{\mathrm{A}}+\mathrm{e}_{\mathrm{A}}=\mathcal{E}_{\mathrm{A}}^{0}+\overline{\mathrm{e}}_{\mathrm{A}}+\mathrm{e}_{\mathrm{A}} \simeq \mathcal{E}_{\mathrm{A}}^{0}+\mathrm{e}_{\mathrm{A}}, \quad \mathcal{B}_{\mathrm{A}}=\overline{\mathcal{B}}_{\mathrm{A}}+\mathrm{b}_{\mathrm{A}} \simeq \mathcal{B}_{\mathrm{A}}^{0}+\mathrm{b}_{\mathrm{A}} \\
& \mathrm{T}_{\mathrm{ai}}=\overline{\mathrm{T}}_{\mathrm{Ai}}+\mathrm{t}_{\mathrm{Ai}}^{*} \simeq \mathrm{T}_{\mathrm{Ai}}^{0}+\mathrm{t}_{\mathrm{Ai}}^{*}, \quad \rho^{R} \mathrm{f}_{\mathrm{i}}=\rho^{R} \overline{\mathrm{F}}_{\mathrm{i}}+\rho^{R} \mathrm{f}_{\mathrm{i}}^{*} \simeq \rho^{R} \mathrm{~F}_{\mathrm{i}}^{0}+\rho^{R} \mathrm{f}_{\mathrm{i}}^{*}
\end{aligned}
$$

where those quantities in rigid body state are denoted by the superscript 0 . The quantities with overbar - are related to $\overline{\mathrm{F}}_{\alpha A}$. The parentheses () in subscript at (17) stands for summation of interchange of indices. In the above derivation, only the fields related to rigid state and mechanical disturbance are relevant. Thus the governing equations (1) and (5) can be decomposed into

$$
\begin{aligned}
& \epsilon_{\mathrm{ABC}} \mathcal{E}_{\mathrm{C}, \mathrm{B}}^{0}+\dot{\mathcal{B}}_{\mathrm{A}}^{0}=0, \quad \mathcal{B}_{\mathrm{A}, \mathrm{A}}^{0}=0, \quad \epsilon_{\mathrm{ABC}} \mathcal{H}_{\mathrm{C}, \mathrm{B}}^{0}=\dot{\mathcal{D}}_{\mathrm{A}}^{0}, \quad \mathcal{D}_{\mathrm{A}, \mathrm{A}}^{0}=0 \\
& \rho^{R} \delta_{\mathrm{iB}} \ddot{\mathrm{X}}_{\mathrm{B}}=\mathrm{T}_{\mathrm{Ai}, \mathrm{A}}^{0}
\end{aligned}
$$

and

$$
\begin{aligned}
& \epsilon_{\mathrm{ABC}} \mathrm{e}_{\mathrm{C}, \mathrm{B}}+\dot{\mathrm{b}}_{\mathrm{A}}=0, \quad \mathrm{~b}_{\mathrm{A}, \mathrm{A}}=0, \quad \epsilon_{\mathrm{ABC}} \mathrm{h}_{\mathrm{C}, \mathrm{B}}=\dot{\mathrm{d}}_{\mathrm{A}}, \quad \mathrm{d}_{\mathrm{A}, \mathrm{A}}=0 \\
& \rho^{R} \delta_{\mathrm{iB}} \ddot{\mathrm{u}}_{\mathrm{B}}=\mathrm{t}_{\mathrm{Ai}, \mathrm{A}}^{*}+\rho^{R} \mathrm{f}_{\mathrm{i}}^{*}
\end{aligned}
$$

The corresponding decompositions of relation (4) and constitutive equation (13) are

$$
\begin{aligned}
& \mathcal{D}_{\mathrm{A}}^{0}= \varepsilon_{0} \mathcal{E}_{\mathrm{A}}^{0}+\mathcal{P}_{\mathrm{A}}^{0}, \quad \mathcal{H}_{\mathrm{A}}^{0}=\frac{1}{\mu_{0}} \mathcal{B}_{\mathrm{A}}^{0}-\epsilon_{\mathrm{ABC}} \dot{\mathrm{X}}_{\mathrm{B}}\left(\varepsilon_{0} \mathcal{E}_{\mathrm{C}}^{0}+\mathcal{P}_{\mathrm{C}}^{0}\right)-\mathcal{M}_{\mathrm{A}}^{0} \\
& \mathcal{P}_{\mathrm{A}}^{0}=\left(\mathrm{P}_{\mathrm{AB}}^{I}+\mathrm{P}_{\mathrm{ABCD}}^{I I} \mathrm{E}_{\mathrm{CD}}^{p}\right) \mathcal{E}_{\mathrm{B}}^{0}, \quad \mathcal{M}_{\mathrm{A}}^{0}=\mathcal{J}_{\mathrm{A}}^{0}=\mathcal{Q}^{0}=\rho^{R} \mathrm{~F}_{\mathrm{i}}^{0}=0 \\
& \mathrm{~T}_{\mathrm{Ai}}^{0}= \delta_{\mathrm{iB}} \mathcal{C}_{\mathrm{ABCD}} \mathrm{E}_{\mathrm{CD}}^{p} \\
& \mathrm{~d}_{\mathrm{A}} \simeq \varepsilon_{0}\left[\mathrm{e}_{\mathrm{A}}+\left(\delta_{\mathrm{AB}} \delta_{\mathrm{CD}}-\delta_{(\mathrm{BC}} \delta_{\mathrm{A}) \mathrm{D}}\right) \mathcal{E}_{\mathrm{B}}^{0} \mathrm{u}_{\mathrm{D}, \mathrm{C}}\right]+\mathrm{p}_{\mathrm{A}} \\
& \mathrm{h}_{\mathrm{A}} \simeq\left\{\frac{1}{\mu_{0}} \mathrm{~b}_{\mathrm{A}}-\epsilon_{\mathrm{ABC}} \dot{\mathrm{X}}_{\mathrm{B}}\left(\varepsilon_{0} \mathrm{e}_{\mathrm{C}}+\mathrm{p}_{\mathrm{C}}\right)+\left[\frac{1}{\mu_{0}} \mathcal{B}_{\mathrm{B}}^{0}-\epsilon_{\mathrm{BCD}} \dot{\mathrm{X}}_{\mathrm{C}}\left(\varepsilon_{0} \mathcal{E}_{\mathrm{D}}^{0}+\mathcal{P}_{\mathrm{D}}^{0}\right)\right]\right. \\
&\left.\left(\mathrm{u}_{(\mathrm{A}, \mathrm{B})}-\delta_{\mathrm{AB}} \mathrm{u}_{\mathrm{E}, \mathrm{E}}\right)-\epsilon_{\mathrm{ACD}}\left(\dot{\mathrm{X}}_{\mathrm{B}} \mathrm{u}_{\mathrm{B}, \mathrm{C}}+\dot{\mathrm{u}}_{\mathrm{C}}\right)\left(\varepsilon_{0} \mathcal{E}_{\mathrm{D}}^{0}+\mathcal{P}_{\mathrm{D}}^{0}\right)\right\} \\
& \mathrm{p}_{\mathrm{A}} \simeq\left(\mathrm{P}_{\mathrm{AB}}^{I}+\mathrm{P}_{\mathrm{ABCD}}^{I I} \mathrm{E}_{\mathrm{CD}}^{p}\right) \mathrm{e}_{\mathrm{B}}+\frac{1}{2} \mathrm{P}_{\mathrm{ABCD}}^{I I} \mathrm{u}_{(\mathrm{C}, \mathrm{D})} \mathcal{E}_{\mathrm{B}}^{0}
\end{aligned}
$$


60 C.-S. Yeh and C.-B. Lin / Mode transition of electromagnetic wave in slab waveguide subjected to in-plane elastic wave

$$
\begin{aligned}
\mathrm{t}_{\mathrm{Ai}}^{*} \simeq & \left.\left(\frac{1}{2} \mathcal{C}_{\mathrm{ABCD}} \mathrm{u}_{(\mathrm{C}, \mathrm{D})}+2 \mathrm{~T}_{\mathrm{AB}}^{I} \mathcal{E}_{\mathrm{C}}^{0} \mathrm{e}_{\mathrm{C}}\right)+\mathrm{P}_{\mathrm{ABCD}}^{I I I} \mathcal{E}_{(\mathrm{C}}^{0} \mathrm{e}_{\mathrm{D})}\right) \delta_{\mathrm{Bi}} \\
+ & \left(\mathcal{C}_{\mathrm{ABCD}} \mathrm{E}_{\mathrm{CD}}^{p}+\mathrm{T}_{\mathrm{AB}}^{I} \mathcal{E}_{\mathrm{C}}^{0} \mathcal{E}_{\mathrm{C}}^{0}+\mathrm{P}_{\mathrm{ABCD}}^{I I I} \mathcal{E}_{\mathrm{C}}^{0} \mathcal{E}_{\mathrm{D}}^{0}\right) \mathrm{u}_{\mathrm{F}, \mathrm{B}} \delta_{\mathrm{iF}} \\
\rho^{R} \mathrm{f}_{\mathrm{i}}^{*}= & {\left[-\left(\mathcal{P}_{\mathrm{B}, \mathrm{B}}^{0} \mathrm{e}_{\mathrm{A}}+\mathrm{p}_{\mathrm{B}, \mathrm{B}} \mathcal{E}_{\mathrm{A}}^{0}\right)+\epsilon_{\mathrm{ABC}}\left(\dot{\mathcal{P}}_{\mathrm{B}}^{0} \mathrm{~b}_{\mathrm{C}}+\dot{\mathrm{p}}_{\mathrm{B}} \mathcal{B}_{\mathrm{C}}^{0}\right)+\left(\mathcal{E}_{\mathrm{A}}^{0} \mathrm{p}_{\mathrm{B}}+\mathrm{e}_{\mathrm{A}} \mathcal{P}_{\mathrm{B}}^{0}\right)_{, \mathrm{B}}\right] \delta_{\mathrm{Ai}} } \\
& +\left[\mathcal{P}_{\mathrm{B}, \mathrm{B}}^{0} \mathcal{E}_{\mathrm{A}}^{0}-\epsilon_{\mathrm{ABC}} \dot{\mathcal{P}}_{\mathrm{B}}^{0} \mathcal{B}_{\mathrm{C}}^{0}+\left(\mathcal{E}_{\mathrm{A}}^{0} \mathcal{P}_{\mathrm{B}}^{0}\right)_{, \mathrm{B}}\right] \delta_{\mathrm{iD}} \mathrm{u}_{\mathrm{A}, \mathrm{D}}
\end{aligned}
$$

Note that the rigid body state approximation has been used in the derivation of above equation.

\section{Jump conditions}

The field quantities or their higher derivatives may suffer discontinuity while across a singular surface. The equations used to describe such phenomena are called jump conditions. Once the singular surface coincides with the boundary of a body, jump conditions become boundary conditions as generally used.

The order of a singular surface depends on that the higher order derivative of position vector jumps across such a surface [8]. For example, the first derivative of position vector (velocity, or deformation gradient, or both) will be discontinuous for the singular surface of order one. The wavefront surfaces of shock waves belong to this catagory. For singular surface of order two, the first derivatives of position vector will be continuous while the second derivatives may suffer jumps. Such a propagating singular surface will be called acceleration wave.

Since the dielectric is a solid whose boundary is regular before deformation rather than after deformation, therefore, the material description is preferred. The governing and constitutive equations have been presented in Ref.14 The general form of corresponding jump conditions across a singular surface can be extended from (5.21) of Ref.8 as

$$
\begin{aligned}
& \epsilon_{\mathrm{ABC}} \llbracket \mathcal{E}_{\mathrm{B}} \rrbracket \mathrm{N}_{\mathrm{C}}+\llbracket \mathcal{B}_{\mathrm{A}} \mathrm{V}^{\Sigma} \rrbracket=0 \\
& \llbracket \mathcal{B}_{\mathrm{A}} \rrbracket \mathrm{N}_{\mathrm{A}}=0 \\
& \epsilon_{\mathrm{ABC}} \llbracket \mathcal{H}_{\mathrm{B}} \rrbracket \mathrm{N}_{\mathrm{C}}-\llbracket \mathcal{D}_{\mathrm{A}} \mathrm{V}^{\Sigma} \rrbracket=\mathcal{J}_{\mathrm{A}}^{\mathrm{S}} \\
& \llbracket \mathcal{D}_{\mathrm{A}} \rrbracket \mathrm{N}_{\mathrm{A}}=\mathcal{Q}^{\mathrm{S}} \\
& \llbracket \mathcal{Q} \mathrm{V}^{\Sigma} \rrbracket-\llbracket \mathcal{J}_{\mathrm{A}} \rrbracket \mathrm{N}_{\mathrm{A}}=0
\end{aligned}
$$

and

$$
\begin{aligned}
& \llbracket \rho^{R} \mathrm{~V}^{\Sigma} \rrbracket=0 \\
& \llbracket \rho^{R} \dot{\mathrm{x}}_{\mathrm{i}} \mathrm{V}^{\Sigma} \rrbracket+\llbracket \mathrm{T}_{\mathrm{Ai}}+\mathrm{T}_{\mathrm{Ai}}^{\mathrm{M}} \rrbracket \mathrm{N}_{\mathrm{A}}=0 \\
& \llbracket\left[\rho^{R}\left(U+\frac{1}{2} \dot{\mathrm{x}}_{\mathrm{i}} \dot{\mathrm{x}}_{\mathrm{i}}\right)-\mathrm{Z}\right] \mathrm{V}^{\Sigma} \rrbracket-\llbracket \dot{\mathrm{x}}_{\mathrm{i}}\left(\mathrm{T}_{\mathrm{iA}}+\mathrm{T}_{\mathrm{i} \mathrm{A}}^{\mathrm{M}}\right)-q_{\mathrm{A}}+\mathrm{Y}_{\mathrm{A}} \rrbracket \mathrm{N}_{\mathrm{A}}=0
\end{aligned}
$$

where

$$
\mathrm{T}_{\mathrm{Ai}}^{\mathrm{M}}=\mathrm{F}_{\mathrm{Bi}}^{-1}\left\{\left(\mathcal{E}_{\mathrm{A}} \mathcal{D}_{\mathrm{B}}+\mathcal{H}_{\mathrm{A}} \mathcal{B}_{\mathrm{B}}\right)-\frac{1}{2} \delta_{\mathrm{AB}}\left[\mathcal{E}_{\mathrm{C}}\left(\mathcal{D}_{\mathrm{C}}-\mathcal{P}_{\mathrm{C}}\right)+\mathcal{B}_{\mathrm{D}}\left(\mathcal{H}_{\mathrm{D}}-\mathcal{M}_{\mathrm{D}}\right)\right]\right\}
$$




$$
\begin{aligned}
& \mathrm{Z}=-\frac{1}{2}\left[\mathcal{E}_{\mathrm{C}}\left(\mathcal{D}_{\mathrm{C}}-\mathcal{P}_{\mathrm{C}}\right)+\mathcal{B}_{\mathrm{D}}\left(\mathcal{H}_{\mathrm{D}}+\mathcal{M}_{\mathrm{D}}\right)\right] \\
& \mathrm{Y}_{\mathrm{A}}=-\epsilon_{\mathrm{ABC}} \mathcal{E}_{\mathrm{B}} \mathcal{H}_{\mathrm{C}}+\mathrm{T}_{\mathrm{iA}}^{\mathrm{M}} \dot{\mathrm{x}}_{\mathrm{i}}
\end{aligned}
$$

These equations are derived under non-relativistic approximation [8]. $\mathrm{V}^{\Sigma}$ and $\mathrm{N}_{\mathrm{A}}$ are speed of propagation and unit normal of the singular surface $\Sigma$ in material description. $\mathcal{J}_{\mathrm{A}}^{\mathrm{S}}, \mathcal{Q}^{\mathrm{S}}$ and $\mathrm{T}_{\mathrm{iA}}^{\mathrm{M}}$ are surface current, surface charge and Maxwell stress, respectively.

The process of applied loading and linearization is similar to that shown in Ref.13. Nevertheless, the jump conditions at the junction of dielectric and vacuum, which will be derived later, are also needed when we deal with the problem of such a case. Since the singular surface considered here is the boundary of dielectric which is fixed under material description, we can specify

$$
\mathcal{J}_{\mathrm{A}}^{\mathrm{S}}=\mathcal{Q}^{\mathrm{S}}=\mathrm{V}^{\Sigma}=0
$$

The vacuum can be viewed as a special medium for electromagnetic waves but not for elastic waves. Therefore, there are no deformations to make the distinction between the spatial and material frames for such a medium. Here we derived the jump conditions of (22a) as an example, other equations needed can be obtained by following the similar procedure and will be listed below. The substitution of (2) into (22a) yields

$$
\epsilon_{\mathrm{ABC}} \llbracket \mathrm{E}_{\mathrm{i}}^{e} \mathrm{~F}_{\mathrm{iB}} \rrbracket \mathrm{N}_{\mathrm{C}}=0
$$

Thereafter, on the basis of decompositions of the deformation gradient, linearization and rigid body state approximation as shown in Ref.13, (26) can be decomposed into two parts with different order of magnitude. Such a decomposition should be exerted in the both side (dielectric and vacuum) of the boundary.

\subsection{Dielectric side}

$$
\mathcal{E}_{\mathrm{B}}^{-}=\mathrm{E}_{\mathrm{i}}^{e^{-}} \mathrm{F}_{\mathrm{iB}}^{-}=\left(\overline{\mathrm{E}}_{\mathrm{i}}^{e^{-}}+\mathrm{e}_{\mathrm{i}}^{e^{-}}\right) \delta_{\mathrm{i} \alpha}\left(\overline{\mathrm{F}}_{\alpha \mathrm{B}}^{-}+\delta_{\alpha \mathrm{D}} \mathrm{u}_{\mathrm{D}, \mathrm{B}}^{-}\right) \simeq \overline{\mathcal{E}}_{\mathrm{B}}^{-}+\mathrm{e}_{\mathrm{B}}^{-}
$$

where

$$
\begin{aligned}
& \overline{\mathcal{E}}_{\mathrm{B}}^{-}=\overline{\mathrm{E}}_{\mathrm{i}}^{e^{-}} \delta_{i \alpha}\left(\delta_{\alpha \mathrm{B}}+\delta_{\alpha \mathrm{E}} \overline{\mathrm{U}}_{\mathrm{E}, \mathrm{B}}^{-}\right) \simeq \mathcal{E}_{\mathrm{B}}^{0^{-}}+\overline{\mathrm{e}}_{\mathrm{B}}^{-} \\
& \mathrm{e}_{\mathrm{B}}^{-}=\overline{\mathrm{E}}_{\mathrm{i}}^{e^{-}} \delta_{\mathrm{iD}} \mathrm{u}_{\mathrm{D}, \mathrm{B}}^{-}+\mathrm{e}_{\mathrm{i}}^{e^{-}} \delta_{\mathrm{i} \alpha} \overline{\mathrm{F}}_{\alpha \mathrm{B}}^{-}
\end{aligned}
$$

and

$$
\begin{aligned}
& \mathcal{E}_{\mathrm{B}}^{0^{-}}=\mathrm{E}_{\mathrm{i}}^{e^{0-}} \delta_{\mathrm{iB}} \\
& \overline{\mathrm{e}}_{\mathrm{B}}^{-}=\mathrm{E}_{\mathrm{i}}^{e^{0-}} \delta_{\mathrm{iE}} \overline{\mathrm{U}}_{\mathrm{E}, \mathrm{B}}^{-}+\overline{\mathrm{e}}_{\mathrm{i}}^{e^{-}} \delta_{\mathrm{i} \alpha} \delta_{\alpha \mathrm{B}}
\end{aligned}
$$

The superscript - stands for that the quantities are inside dielectric. Those field quantities with overbar - and superscript 0 are those in the intermediate state and rigid body state [13], respectively. The decomposition of $\mathrm{E}_{\mathrm{i}}^{e^{-}}$can be written as

$$
\begin{aligned}
\mathrm{E}_{\mathrm{i}}^{e^{-}} & =\mathrm{E}_{\mathrm{i}}^{-}+\epsilon_{\mathrm{ijk}} \dot{\mathrm{x}}_{\mathrm{j}}^{-} \mathrm{B}_{\mathrm{k}}=\left(\mathrm{E}_{\mathrm{i}}^{-}+\mathrm{e}_{\mathrm{i}}^{-}\right)+\epsilon_{\mathrm{ijk}} \delta_{\mathrm{j} \alpha}\left(\dot{\xi}_{\alpha}^{-}+\delta_{\alpha \mathrm{A}} \dot{\mathrm{u}}_{\mathrm{A}}^{-}\right) \\
& =\left(\delta_{\mathrm{k} \alpha} \mathrm{B}_{\alpha}^{-}+\mathrm{b}_{\mathrm{k}}^{-}\right) \simeq \overline{\mathrm{E}}_{\mathrm{i}}^{e^{-}}+\mathrm{e}_{\mathrm{i}}^{e^{-}}
\end{aligned}
$$


where

$$
\begin{aligned}
\overline{\mathrm{E}}_{\mathrm{i}}^{e^{-}} & =\overline{\mathrm{E}}_{\mathrm{i}}^{-}+\epsilon_{\mathrm{ijk}} \delta_{\mathrm{j} \alpha} \dot{\xi}_{\alpha}^{-} \delta_{\mathrm{k} \beta} \mathrm{B}_{\beta}^{-} \\
& =\left(\mathrm{E}_{\mathrm{i}}^{0-}+\overline{\mathrm{e}}_{\mathrm{i}}^{-}\right)+\epsilon_{\mathrm{ijk}} \delta_{\mathrm{jB}} \dot{\overline{\mathrm{U}}}_{\mathrm{B}}^{-} \delta_{\mathrm{k} \beta}\left(\delta_{\beta \mathrm{K}} \mathrm{B}_{\mathrm{K}}^{0-}+\overline{\mathrm{b}}_{\beta}^{-}\right) \simeq \mathrm{E}_{\mathrm{i}}^{0-}+\overline{\mathrm{e}}_{\mathrm{i}}^{e^{-}} \\
\mathrm{e}_{\mathrm{i}}^{e^{-}} & =\mathrm{e}_{\mathrm{i}}^{-}+\epsilon_{\mathrm{ijk}} \delta_{\mathrm{j} \alpha}\left(\dot{\xi}_{\alpha}^{-} \mathrm{b}_{\mathrm{k}}^{-}+\delta_{\alpha \mathrm{A}} \dot{\mathrm{u}}_{\mathrm{A}}^{-} \delta_{\mathrm{k} \beta} \mathrm{B}_{\beta}^{-}\right) \\
\overline{\mathrm{e}}_{\mathrm{i}}^{e^{-}} & =\overline{\mathrm{e}}_{\mathrm{i}}^{-}+\epsilon_{\mathrm{ijk}} \delta_{\mathrm{jB}} \dot{\overline{\mathrm{U}}}_{\mathrm{B}}^{-} \delta_{\mathrm{kC}} \mathrm{B}_{\mathrm{C}}^{0-}
\end{aligned}
$$

Note that the static feature of $\mathrm{X}_{\mathrm{A}}$ has been used in the derivations of $(27) \sim(31)$. Through the linearization and rigid body state approximation ${ }^{14}$ and the estimations

$$
\begin{aligned}
& \frac{\epsilon_{\mathrm{ijk}} \delta_{\mathrm{j} \alpha} \dot{\xi}_{\alpha}^{-} \mathrm{b}_{\mathrm{k}}^{-}}{\mathrm{e}_{\mathrm{i}}^{-}} \sim \frac{\dot{\overline{\mathrm{U}}}_{\mathrm{A}}\left|\frac{K}{\Omega} \mathrm{e}_{\mathrm{k}}^{-}\right|}{\left|\mathrm{e}_{\mathrm{i}}^{-}\right|} \sim \Omega \overline{\mathrm{U}}_{\mathrm{A}} \frac{K}{\Omega} \sim \overline{\mathrm{U}}_{\mathrm{A}, \mathrm{B}} \ll 1 \\
& \frac{\epsilon_{\mathrm{ijk}} \delta_{\mathrm{jA}} \dot{\mathrm{u}}_{\mathrm{A}}^{-} \delta_{\mathrm{k} \alpha} \overline{\mathrm{B}}_{\alpha}^{-}}{\mathrm{e}_{\mathrm{i}}^{-}} \sim \frac{\dot{\mathrm{u}}_{\mathrm{A}}^{-}\left|\frac{K}{\Omega} \overline{\mathrm{E}}_{\beta}^{-}\right|}{\left|\mathrm{u}_{\mathrm{A}, \mathrm{B}}^{-} \overline{\mathrm{E}}_{\gamma}^{-}\right|} \sim \frac{\omega \mathrm{u}_{\mathrm{A}}^{-} \frac{K}{\Omega}}{k_{\mathrm{B}} \mathrm{u}_{\mathrm{A}}^{-}} \sim \frac{\omega / k_{\mathrm{B}}}{\Omega / K} \simeq \frac{c_{e}}{c} \ll 1
\end{aligned}
$$

we can obtain

$$
\mathcal{E}_{\mathrm{B}}^{-}=\mathrm{E}_{\mathrm{i}}^{e^{-}} \mathrm{F}_{\mathrm{iA}}^{-} \simeq \mathcal{E}_{\mathrm{B}}^{0-}+\mathrm{e}_{\mathrm{B}}^{-}
$$

where

$$
\mathcal{E}_{\mathrm{B}}^{0-}=\mathrm{E}_{\mathrm{i}}^{0-} \delta_{\mathrm{iB}}, \quad \mathrm{e}_{\mathrm{B}}^{-}=\delta_{\mathrm{iD}}\left(\mathrm{E}_{\mathrm{i}}^{0-} \mathrm{u}_{\mathrm{D}, \mathrm{B}}^{-}+\mathrm{e}_{\mathrm{i}}^{-} \delta_{\mathrm{BD}}\right)
$$

\subsection{Vacuum side}

$$
\begin{aligned}
\mathrm{E}_{\mathrm{i}}^{e^{+}} \mathrm{F}_{\mathrm{iA}}^{-} & =\mathrm{E}_{\mathrm{i}}^{+} \mathrm{F}_{\mathrm{iA}}^{-}=\left(\overline{\mathrm{E}}_{\mathrm{i}}^{+}+\mathrm{e}_{\mathrm{i}}^{+}\right) \delta_{\mathrm{i} \alpha}\left(\overline{\mathrm{F}}_{\alpha \mathrm{A}}^{-}+\delta_{\alpha \mathrm{D}} \mathrm{u}_{\mathrm{D}, \mathrm{B}}^{-}\right) \\
& \simeq\left(\overline{\mathrm{E}}_{\mathrm{i}}^{+}+\overline{\mathrm{e}}_{\mathrm{i}}^{+}+\mathrm{e}_{\mathrm{i}}^{+}\right) \delta_{\mathrm{i} \alpha}\left(\delta_{\alpha \mathrm{A}}+\delta_{\alpha \mathrm{C}} \overline{\mathrm{U}}_{\mathrm{C}, \mathrm{B}}^{-}+\delta_{\alpha \mathrm{D}} \mathrm{u}_{\mathrm{D}, \mathrm{B}}^{-}\right) \simeq \mathrm{E}_{\mathrm{B}}^{0+}+\mathrm{e}_{\mathrm{B}}^{+}
\end{aligned}
$$

where

$$
\mathrm{E}_{\mathrm{B}}^{0+}=\mathrm{E}_{\mathrm{i}}^{0+} \delta_{\mathrm{iB}}, \quad \mathrm{e}_{\mathrm{B}}^{+}=\mathrm{e}_{\mathrm{i}}^{+} \delta_{\mathrm{iB}}+\mathrm{E}_{\mathrm{i}}^{0+} \delta_{\mathrm{iD}} \mathrm{u}_{\mathrm{D}, \mathrm{B}}^{-}
$$

The superscript + stands for the quantities in the vacuum side. The deformation gradient with superscript - represents that the deformation in the boundary of dielectric side is used to time the fields in vacuum side artifically for the sake of compatibility in material configuration. Note that the replacement of $\mathrm{E}_{\mathrm{i}}^{e^{+}}$ by $\mathrm{E}_{\mathrm{i}}^{+}$is adopted in the derivation of (35) which is also due to the absence of deformation in vacuum.

Since the fields in vacuum must be functions of spatial coordinate $\mathrm{x}_{\mathrm{i}}$, they can be written as functions of material coordinate $\mathrm{X}_{\mathrm{A}}$ by the following expansion

$$
\mathrm{E}_{\mathrm{i}}^{0+}=\hat{\mathrm{E}}_{\mathrm{i}}^{0+}\left(\mathrm{x}_{\mathrm{j}}\right)=\hat{\mathrm{E}}_{\mathrm{i}}^{0+}\left(\delta_{\mathrm{jL}}\left[\mathrm{X}_{\mathrm{L}}+\overline{\mathrm{U}}_{\mathrm{L}}^{-}+\mathrm{u}_{\mathrm{L}}^{-}\right]\right) \simeq \hat{\mathrm{E}}_{\mathrm{i}}^{0+}\left(\delta_{\mathrm{jL}} \mathrm{X}_{\mathrm{L}}\right)+\left.\frac{\partial \hat{\mathrm{E}}_{\mathrm{i}}^{0+}}{\partial \mathrm{x}_{\mathrm{j}}}\right|_{\mathrm{x}_{\mathrm{j}}=\delta_{\mathrm{jL}} \mathrm{X}_{\mathrm{L}}} \delta_{\mathrm{jE}} \mathrm{u}_{\mathrm{E}}^{-}
$$

similarly,

$$
\mathrm{e}_{\mathrm{i}}^{+}=\left.\hat{\mathrm{e}}_{\mathrm{i}}^{+}\left(\mathrm{x}_{\mathrm{j}}\right) \simeq \hat{\mathrm{e}}_{\mathrm{i}}^{+}\left(\delta_{\mathrm{jL}} \mathrm{X}_{\mathrm{L}}\right) \frac{\partial \hat{\mathrm{e}}_{\mathrm{i}}^{+}}{\partial \mathrm{x}_{\mathrm{j}}}\right|_{\mathrm{x}_{\mathrm{j}}=\delta_{\mathrm{j} \mathrm{L}} \mathrm{X}_{\mathrm{L}}}+\delta_{\mathrm{jD}} \mathrm{u}_{\mathrm{D}}^{-}
$$


The approximation $\left|\overline{\mathrm{U}}_{\mathrm{B}}\right| \ll\left|\mathrm{u}_{\mathrm{B}}\right|$ used here is derived from

$$
\left|\overline{\mathrm{U}}_{\mathrm{A}, \mathrm{B}}\right| \sim\left|K_{\mathrm{B}} \overline{\mathrm{U}}_{\mathrm{A}}\right| \ll\left|\mathrm{u}_{\mathrm{A}, \mathrm{B}}\right| \sim\left|k_{\mathrm{B}} \mathrm{u}_{\mathrm{A}}\right|
$$

and the estimation $k \ll K^{14}$

From (27), (28), (29) and (35), (26) can be decomposed as

$$
\epsilon_{\mathrm{ABC}} \mathcal{E}_{\mathrm{B}}^{0-} \mathrm{N}_{\mathrm{C}}=\epsilon_{\mathrm{ABC}} \mathrm{E}_{\mathrm{B}}^{0+} \mathrm{N}_{\mathrm{C}}
$$

and

$$
\epsilon_{\mathrm{ABC}} \mathrm{e}_{\mathrm{B}}^{-} \mathrm{N}_{\mathrm{C}}=\epsilon_{\mathrm{ABC}}\left(\mathrm{e}_{\mathrm{B}}^{+}+\mathrm{E}_{\mathrm{Bj}}^{0 \prime} \delta_{\mathrm{jE}} \mathrm{u}_{\mathrm{E}}^{-}+\mathrm{E}_{\mathrm{D}}^{0+} \mathrm{u}_{\mathrm{D}, \mathrm{B}}^{-}\right) \mathrm{N}_{\mathrm{C}}
$$

where

$$
\mathrm{E}_{\mathrm{B}}^{0+}=\delta_{\mathrm{Bi}} \hat{\mathrm{E}}_{\mathrm{i}}^{0+}\left(\delta_{\mathrm{jL}} \mathrm{X}_{\mathrm{L}}\right), \quad \mathrm{e}_{\mathrm{B}}^{+}=\delta_{\mathrm{Bi}} \hat{\mathrm{e}}_{\mathrm{i}}^{+}\left(\delta_{\mathrm{jL}} \mathrm{X}_{\mathrm{L}}\right), \quad \mathrm{E}_{\mathrm{Bj}}^{0 \prime}=\left.\delta_{\mathrm{Bi}} \frac{\partial \hat{\mathrm{E}}_{\mathrm{i}}^{0+}}{\partial \mathrm{x}_{\mathrm{j}}}\right|_{\mathrm{x}_{\mathrm{j}}=\delta_{\mathrm{jL}} \mathrm{X}_{\mathrm{L}}}
$$

Similarly, the other equations in (22) can also be decomposed into

$$
\begin{aligned}
& \mathcal{B}_{\mathrm{A}}^{0-} \mathrm{N}_{\mathrm{A}}=\mathrm{B}_{\mathrm{A}}^{0+} \mathrm{N}_{\mathrm{A}} \\
& \epsilon_{\mathrm{ABC}} \mathcal{H}_{\mathrm{B}}^{0-} \mathrm{N}_{\mathrm{C}}=\epsilon_{\mathrm{ABC}}\left(\frac{1}{\mu_{0}} \mathrm{~B}_{\mathrm{B}}^{0+}\right) \mathrm{N}_{\mathrm{C}} \\
& \mathcal{D}_{\mathrm{A}}^{0-} \mathrm{N}_{\mathrm{A}}=\left(\varepsilon_{0} \mathrm{E}_{\mathrm{A}}^{0+}\right) \mathrm{N}_{\mathrm{A}} \\
& \mathrm{T}_{\mathrm{Ai}}^{0-} \mathrm{N}_{\mathrm{A}}=0
\end{aligned}
$$

and

$$
\begin{aligned}
& \mathrm{b}_{\mathrm{A}}^{-} \mathrm{N}_{\mathrm{A}}=\left[\mathrm{b}_{\mathrm{A}}^{+}+\mathrm{B}_{\mathrm{Aj}}^{0 \prime} \delta_{\mathrm{j} D} \mathrm{u}_{\mathrm{D}}^{-}+\left(\delta_{\mathrm{AC}} \mathrm{u}_{\mathrm{B}, \mathrm{B}}^{-} \mathrm{u}_{\mathrm{A}, \mathrm{C}}^{-}\right) \mathrm{B}_{\mathrm{C}}^{0+}\right] \mathrm{N}_{\mathrm{A}} \\
& \epsilon_{\mathrm{ABC}} \mathrm{h}_{\mathrm{B}}^{-} \mathrm{N}_{\mathrm{C}}=\epsilon_{\mathrm{ABC}} \frac{1}{\mu_{0}}\left(\mathrm{~b}_{\mathrm{B}}^{+}+\mathrm{B}_{\mathrm{Bj}}^{0 \prime} \delta_{\mathrm{jE}} \mathrm{u}_{\mathrm{E}}^{-}+\mathrm{B}_{\mathrm{D}}^{0+} \mathrm{u}_{\mathrm{D}, \mathrm{B}}^{-}\right) \mathrm{N}_{\mathrm{C}} \\
& \mathrm{d}_{\mathrm{A}}^{-} \mathrm{N}_{\mathrm{A}}=\varepsilon_{0}\left[\mathrm{e}_{\mathrm{A}}^{+}+\mathrm{E}_{\mathrm{Aj}}^{0 \prime} \delta_{\mathrm{jD}} \mathrm{u}_{\mathrm{D}}^{-}+\left(\delta_{\mathrm{AC}} \mathrm{u}_{\mathrm{B}, \mathrm{B}}^{-} \mathrm{u}_{\mathrm{A}, \mathrm{C}}^{-}\right) \mathrm{E}_{\mathrm{C}}^{0+}\right] \mathrm{N}_{\mathrm{A}} \\
& \left(\mathrm{t}_{\mathrm{Ai}}^{*-}+\mathrm{t}_{\mathrm{Ai}}^{\mathrm{M}^{*-}}\right) \mathrm{N}_{\mathrm{A}}=\left(\mathrm{t}_{\mathrm{Ai}}^{\mathrm{M}^{*+}}+\mathrm{t}_{\mathrm{Aik}}^{\mathrm{M}^{0 \prime}} \delta_{\mathrm{kE}} \mathrm{u}_{\mathrm{E}}^{-}\right)
\end{aligned}
$$

where

$$
\begin{aligned}
\mathrm{t}_{\mathrm{Ai}}^{\mathrm{M}^{*-}}= & \delta_{\mathrm{Bi}}\left\{\left(\mathcal{E}_{\mathrm{A}}^{0-} \mathrm{d}_{\mathrm{B}}^{-}+\mathrm{e}_{\mathrm{A}}^{-} \mathcal{D}_{\mathrm{B}}^{0-}+\mathcal{H}_{\mathrm{A}}^{0-} \mathrm{b}_{\mathrm{B}}^{-}+\mathrm{h}_{\mathrm{A}}^{-} \mathcal{B}_{\mathrm{B}}^{0-}\right)-\frac{1}{2} \delta_{\mathrm{AB}}\left[\mathcal{E}_{\mathrm{C}}^{0-}\left(\mathrm{d}_{\mathrm{C}}^{-}-\mathrm{p}_{\mathrm{C}}^{-}\right)\right.\right. \\
& \left.\left.+\mathrm{e}_{\mathrm{C}}^{-}\left(\mathcal{D}_{\mathrm{C}}^{0-}-\mathcal{P}_{\mathrm{C}}^{0-}\right)+\mathrm{b}_{\mathrm{D}}^{-}\left(\mathcal{H}_{\mathrm{D}}^{0-}-\mathcal{M}_{\mathrm{D}}^{0-}\right)+\mathcal{B}_{\mathrm{D}}^{0-}\left(\mathrm{h}_{\mathrm{D}}^{-}-\mathrm{m}_{\mathrm{D}}^{-}\right)\right]\right\}-\mathrm{u}_{\mathrm{B}, \mathrm{D}}^{-} \delta_{\mathrm{Di}} \\
& \left\{\left(\mathcal{E}_{\mathrm{A}}^{0-} \mathcal{D}_{\mathrm{B}}^{0-}+\mathcal{H}_{\mathrm{A}}^{0-} \mathcal{B}_{\mathrm{B}}^{0-}\right)-\frac{1}{2} \delta_{\mathrm{AB}}\left[\mathcal{E}_{\mathrm{C}}^{0-}\left(\mathcal{D}_{\mathrm{C}}^{0-}-\mathcal{P}_{\mathrm{C}}^{0-}\right)+\mathcal{B}_{\mathrm{D}}^{0-}\left(\mathcal{H}_{\mathrm{D}}^{0-}-\mathcal{M}_{\mathrm{D}}^{0-}\right]\right\}\right. \\
\mathrm{t}_{\mathrm{Aik}}^{\mathrm{M}^{0 \prime}}= & \left.\delta_{\mathrm{Aj}} \frac{\partial \hat{\mathrm{t}}_{\mathrm{ji}}^{\mathrm{M}^{0+}}}{\partial \mathrm{x}_{\mathrm{k}}}\right|_{\mathrm{x}_{\mathrm{k}}=\delta_{\mathrm{kL}} \mathrm{X}_{\mathrm{L}}}
\end{aligned}
$$

The definitions for various notations of magnetic flux in vacuum side $\left(\mathrm{B}_{\mathrm{A}}^{0+}, \mathrm{b}_{\mathrm{A}}^{+}, \mathrm{B}_{\mathrm{Bj}}^{0 \prime}\right)$ follow the similar form as those of electric fields shown in (41). It is worth to mention here that the Maxwell stresses in terms of the electromagnetic fields in rigid body state can be negligible in both sides of (43d) since they should appear in the boundary conditions of the displacement $\vec{U}_{A}$ which is defined in (16). 
64 C.-S. Yeh and C.-B. Lin / Mode transition of electromagnetic wave in slab waveguide subjected to in-plane elastic wave

\section{The alternation of electromagnetic wave in slab waveguide}

The equivalence between deformable electrodynamics and photoelastic theory for the phase change of electromagnetic wave in an infinite medium has been shown in Ref.14 and 15. Nevertheless, since the boundary conditions derived in the previous section are valid only for deformable electrodynamic theory, the photoelastic theory is discarded here. For convenience, the slab waveguide which is surrounded by the vacuum has been placed in $\mathrm{X}_{1}-\mathrm{X}_{3}$ plane and occupy the region $\left|\mathrm{X}_{2}\right|<\frac{\mathrm{d}}{2}$.

The electromagnetic fields in rigid body state should be solved preliminarily. By making use of (18), (20), (40a) and (42) with the convenient choice of field direction, a series of discrete guided modes for electromagnetic wave propagating in slab waveguide can be obtained [17-19]. The characteristic equations for guided TE and TM modes will be presented in Table 1 [18], where the corresponding electromagnetic fields and cut-off frequency for each type of discrete guided mode are also listed.

Table 1

The field distribution and some characteristic data of guided TE mode

\begin{tabular}{|c|c|c|}
\hline TE mode & odd & even \\
\hline $\begin{array}{l}\text { characteristic eq. } \\
\text { cut-off frequency }\end{array}$ & $\begin{array}{c}\frac{\alpha}{K_{2}}=\tan \frac{K_{2} \mathrm{~d}}{2} \\
(\mathrm{~m}-1) / \mathrm{M} \quad(\mathrm{m}=1,2, \cdots)\end{array}$ & $\begin{array}{c}\frac{\alpha}{K_{2}}=-\cot \frac{K_{2} \mathrm{~d}}{2} \\
\left(\mathrm{~m}-\frac{1}{2}\right) / \mathrm{M} \quad(\mathrm{m}=1,2, \cdots)\end{array}$ \\
\hline$\left|X_{2}\right|<\frac{d}{2}$ & $\begin{array}{c}\mathcal{E}_{3}^{0^{-}}=E_{3} \mathrm{C}_{2} \mathrm{C}_{1} \\
\mathcal{B}_{1}^{0^{-}}=-\frac{K_{2}}{\Omega} E_{3} \mathrm{~S}_{2} \mathrm{~S}_{1} \\
\mathcal{B}_{2}^{0^{-}}=-\frac{K_{1}}{\Omega} E_{3} \mathrm{C}_{2} \mathrm{C}_{1}\end{array}$ & $\begin{array}{c}\mathcal{E}_{3}^{0^{-}}=E_{3} \mathrm{~S}_{2} \mathrm{C}_{1} \\
\mathcal{B}_{1}^{0^{-}}=\frac{K_{2}}{\Omega} E_{3} \mathrm{C}_{2} \mathrm{~S}_{1} \\
\mathcal{B}_{2}^{0^{-}}=-\frac{K_{1}}{\Omega} E_{3} \mathrm{~S}_{2} \mathrm{C}_{1}\end{array}$ \\
\hline$X_{2}>\frac{d}{2}$ & $\begin{array}{c}\mathrm{E}_{3}^{0^{+}}=E_{3}^{\mathrm{C}} \mathrm{D}_{2} \mathrm{C}_{1} \\
\mathrm{~B}_{1}^{0^{+}}=-\frac{\alpha}{\Omega} E_{3}^{\mathrm{C}} \mathrm{D}_{2} \mathrm{~S}_{1} \\
\mathrm{~B}_{2}^{0^{+}}=-\frac{K_{1}}{\Omega} E_{3}^{\mathrm{C}} \mathrm{D}_{2} \mathrm{C}_{1}\end{array}$ & $\begin{array}{c}\mathrm{E}_{3}^{0^{+}}=E_{3}^{\mathrm{S}} \mathrm{D}_{2} \mathrm{C}_{1} \\
\mathrm{~B}_{1}^{0^{+}}=-\frac{\alpha}{\Omega} E_{3}^{\mathrm{S}} \mathrm{D}_{2} \mathrm{~S}_{1} \\
\mathrm{~B}_{2}^{0^{+}}=-\frac{K_{1}}{\Omega} E_{3}^{\mathrm{S}} \mathrm{D}_{2} \mathrm{C}_{1}\end{array}$ \\
\hline $\mathrm{X}_{2}<-\frac{\mathrm{d}}{2}$ & $\begin{array}{c}\mathrm{E}_{3}^{0^{+}}=E_{3}^{\mathrm{C}} \mathrm{D}_{2} \mathrm{C}_{1} \\
\mathrm{~B}_{1}^{0^{+}}=\frac{\alpha}{\Omega} E_{3}^{\mathrm{C}} \mathrm{D}_{2}^{*} \mathrm{~S}_{1} \\
\mathrm{~B}_{2}^{0^{+}}=-\frac{K_{1}}{\Omega} E_{3}^{\mathrm{C}} \mathrm{D}_{2}^{*} \mathrm{C}_{1}\end{array}$ & $\begin{array}{c}\mathrm{E}_{3}^{0^{+}}=E_{3}^{\mathrm{S}} \mathrm{D}_{2}^{*} \mathrm{C}_{1} \\
\mathrm{~B}_{1}^{0^{+}}=-\frac{\alpha}{\Omega} E_{3}^{\mathrm{S}} \mathrm{D}_{2}^{*} \mathrm{~S}_{1} \\
\mathrm{~B}_{2}^{0^{+}}=\frac{K_{1}}{\Omega} E_{3}^{\mathrm{S}} \mathrm{D}_{2}^{*} \mathrm{C}_{1}\end{array}$ \\
\hline
\end{tabular}

where

$$
\begin{aligned}
& \mathrm{M}=\mathrm{d} \sqrt{\mu_{0}\left(\varepsilon_{\mathrm{d}}-\varepsilon_{0}\right)} \\
& \mathrm{C}_{1}=\cos \left(K_{1} \mathrm{X}_{1}-\Omega \mathrm{t}+\Phi_{0}\right), \quad \mathrm{C}_{2}=2 \cos K_{2} \mathrm{X}_{2} \\
& \mathrm{~S}_{1}=\sin \left(K_{1} \mathrm{X}_{1}-\Omega \mathrm{t}+\Phi_{0}\right), \quad \mathrm{S}_{2}=2 \sin K_{2} \mathrm{X}_{2} \\
& \mathrm{D}_{2}=2 \exp \left[-\alpha\left(\mathrm{X}_{2}-\frac{\mathrm{d}}{2}\right)\right], \quad \mathrm{D}_{2}^{*}=2 \exp \left[\alpha\left(\mathrm{X}_{2}+\frac{\mathrm{d}}{2}\right)\right] \\
& E_{3}^{\mathrm{C}}=E_{3} \cos \frac{K_{2} \mathrm{~d}}{2}, \quad E_{3}^{\mathrm{S}}=E_{3} \sin \frac{K_{2} \mathrm{~d}}{2} \\
& K_{2}=\left(\mu_{0} \varepsilon_{\mathrm{d}} \Omega^{2}-K_{1}^{2}\right)^{\frac{1}{2}}, \quad \alpha=\left(K_{1}^{2}-\mu_{0} \varepsilon_{0} \Omega^{2}\right)^{\frac{1}{2}}
\end{aligned}
$$

In Table 1, the cut-off frequency is the lowest bound for each mode of propagating electromagnetic wave. This means that each propagating mode becomes evanescent when its frequency is lower than the corresponding cut-off frequency. For convenience, only the lowest TE mode is considered here. 
Nevertheless, the same procedure introduced here can also be applied to study the higher TE or TM modes. For optically isotropic dielectric slab waveguide, the electric permittivity $\varepsilon_{\mathrm{d}}$ can be denoted as

$$
\varepsilon_{\mathrm{d}}=\varepsilon_{11}=\varepsilon_{22}=\varepsilon_{33}=\varepsilon_{0} \mathrm{n}^{2}
$$

The perturbed displacement and electromagnetic fields can be approximated by the lowest order [14] i.e.

$$
\mathrm{u}_{\mathrm{A}}^{-} \simeq \mathrm{u}_{\mathrm{A}}^{(0)}, \quad \mathrm{e}_{\mathrm{A}}^{-} \simeq \mathrm{e}_{\mathrm{A}}^{(1)}, \quad \mathrm{b}_{\mathrm{A}}^{-} \simeq \mathrm{b}_{\mathrm{A}}^{(1)}
$$

Therefore, it is only needed to find the lowest order perturbed fields $u_{A}^{(0)}$ and $e_{A}^{(1)}$ sequentially. It is assumed that the dielectric is isotropic in material state while the predeformation is absent. The displacement $u_{\mathrm{A}}^{(0)}$ satisfies

$$
\begin{aligned}
& \rho^{R} \ddot{\mathrm{u}}_{\mathrm{B}}^{(0)}=\tau_{\mathrm{AB}, \mathrm{A}} \\
& \tau_{\mathrm{AB}} \mathrm{N}_{\mathrm{A}}=0
\end{aligned}
$$

where

$$
\tau_{A B}=\lambda \delta_{A B} \mathrm{u}_{\mathrm{D}, \mathrm{D}}+\mu\left(\mathrm{u}_{\mathrm{A}, \mathrm{B}}+\mathrm{u}_{\mathrm{B}, \mathrm{A}}\right)
$$

\begin{tabular}{|c|c|c|}
\hline mode & symmetric & anti-symmetric \\
\hline characteristic eq. & $\frac{\tan \left(k_{2}^{\mathrm{T}} \mathrm{d} / 2\right)}{\tan \left(k_{2}^{\mathrm{L}} \mathrm{d} / 2\right)}=\mathrm{H}$ & $\frac{\tan \left(k_{2}^{\mathrm{T}} \mathrm{d} / 2\right)}{\tan \left(k_{2}^{\mathrm{L}} \mathrm{d} / 2\right)}=\frac{1}{\mathrm{H}}$ \\
\hline displacement & $\sum_{\mathrm{a}=\mathrm{I}}^{\mathrm{IV}} u_{\mathrm{P}}^{\mathrm{a}} \exp \left(i \xi^{\mathrm{a}}\right) \mathrm{P}=1,2$ & $\sum_{\mathrm{a}=\mathrm{I}}^{\mathrm{IV}} u_{\mathrm{P}}^{\mathrm{a}} \exp \left(i \xi^{\mathrm{a}}\right) \mathrm{P}=1,2$ \\
\hline amplitude & $\begin{array}{c}u_{\mathrm{P}}^{\mathrm{I}, \mathrm{II}}=-u^{\mathrm{L}}\left(k_{1}^{\mathrm{a}}, k_{2}^{\mathrm{a}}, 0\right) \\
u_{\mathrm{P}}^{\mathrm{III}}=u^{\mathrm{T}}\left(k_{2}^{\mathrm{a}},-k_{1}^{\mathrm{a}}, 0\right) \\
u_{\mathrm{P}}^{\mathrm{IV}}=-u^{\mathrm{T}}\left(k_{2}^{\mathrm{a}},-k_{1}^{\mathrm{a}}, 0\right)\end{array}$ & $\begin{array}{c}u_{\mathrm{P}}^{\mathrm{I}}=u^{\mathrm{L}}\left(k_{1}^{\mathrm{a}}, k_{2}^{\mathrm{a}}, 0\right) \\
u_{\mathrm{P}}^{\mathrm{II}}=-u^{\mathrm{L}}\left(k_{1}^{\mathrm{a}}, k_{2}^{\mathrm{a}}, 0\right) \\
u_{\mathrm{P}}^{\mathrm{III}, \mathrm{IV}}=-u^{\mathrm{T}}\left(k_{2}^{\mathrm{a}},-k_{1}^{\mathrm{a}}, 0\right)\end{array}$ \\
\hline phase & $\xi^{\mathrm{a}}=k_{P} \mathrm{X}_{P}-\omega \mathrm{t}+\varphi^{\mathrm{a}_{0}}$ & $\xi^{\mathrm{a}}=k_{P} \mathrm{X}_{P}-\omega \mathrm{t}+\varphi^{\mathrm{a}_{0}}$ \\
\hline relations of phase & 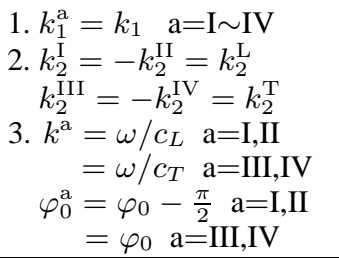 & 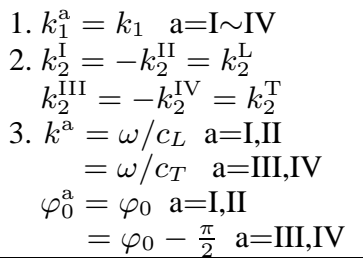 \\
\hline
\end{tabular}

Since the dielectric slab is a waveguide for elastic waves also, the solution of (48) can be characterized by the discrete propagating mode listed in Table 2 [20].

Table 2

The displacement field for mode of in-plane elastic wave

where

$$
\mathrm{H}=-\frac{4 k_{1}^{2} k_{2}^{\mathrm{L}} k_{2}^{\mathrm{T}}}{\left(k^{\mathrm{T}^{2}}-k_{1}^{2}\right)}
$$


The characteristic equations which are called the Rayleigh-Lamb frequency equations can also be rewritten as

$$
\begin{array}{ll}
\frac{\tan \left[\frac{\pi}{2}\left(\bar{\omega}^{2}-\xi^{2}\right)^{\frac{1}{2}}\right]}{\tan \left[\frac{\pi}{2}\left(\frac{\bar{\omega}^{2}}{\kappa^{2}}-\xi^{2}\right)^{\frac{1}{2}}\right]}=\mathrm{H} & \text { symmetric } \\
\frac{\tan \left[\frac{\pi}{2}\left(\bar{\omega}^{2}-\xi^{2}\right)^{\frac{1}{2}}\right]}{\tan \left[\frac{\pi}{2}\left(\frac{\bar{\omega}^{2}}{\kappa^{2}}-\xi^{2}\right)^{\frac{1}{2}}\right]}=\frac{1}{\mathrm{H}} & \text { antisymmetric }
\end{array}
$$

where

$$
\begin{aligned}
& \mathrm{H}=\hat{\mathrm{H}}(\xi, \bar{\omega})=-\frac{4 \xi^{2}\left(\frac{\bar{\omega}^{2}}{\kappa^{2}}-\xi^{2}\right)^{\frac{1}{2}}\left(\bar{\omega}^{2}-\xi^{2}\right)^{\frac{1}{2}}}{\bar{\omega}^{2}-2 \xi^{2}} \\
& \xi=\frac{k_{1} \mathrm{~d}}{\pi}, \quad \bar{\omega}=\frac{\omega \mathrm{d}}{\pi c_{T}}, \quad \kappa=\frac{c_{L}}{c_{T}}
\end{aligned}
$$

The solutions of (51) can be plotted as series of branch in $\xi-\bar{\omega}$ plane. Each branch stands for a propagation mode [20]. Just like the feature of cut-off frequency for the electromagnetic wave, each mode of elastic wave becomes non-propagating once its frequency is lower than its cut-off frequency.

Now we turn to solve the perturbed electromagnetic fields. On the basis of Ref.14 and the displacement fields listed above, the perturbed electric field $\mathrm{e}^{(1)}$ satisfies

$$
\mathrm{e}_{\mathrm{B}, \mathrm{BA}}^{(1)}-\mathrm{e}_{\mathrm{A}, \mathrm{BB}}^{(1)}+\mu_{0} \varepsilon(\mathrm{A})(\mathrm{A}) \ddot{\mathrm{e}}_{\mathrm{I}}^{(1)}=\sum_{+,-} \sum_{\mathrm{a}=\mathrm{I}}^{\mathrm{IV}} \Re e\left[\Lambda_{\mathrm{A} \pm}^{\mathrm{a}} \exp \left(i \Phi_{ \pm}^{\mathrm{a}}\right)+\Lambda_{\mathrm{A} \pm}^{\mathrm{Ra}} \exp \left(i \Phi_{ \pm}^{\mathrm{Ra}}\right)\right]
$$

where

$$
\begin{aligned}
\Lambda_{1 \pm}^{\mathrm{a}}= & \Lambda_{2 \pm}^{\mathrm{a}}=\Lambda_{1 \pm}^{\mathrm{Ra}}=\Lambda_{2 \pm}^{\mathrm{Ra}}=0 \\
\Lambda_{3 \pm}^{\mathrm{a}}= & \frac{1}{\Omega}\left[K_{2}\left(K_{1} \pm k_{1}\right)+K_{1}\left(K_{2} \pm k_{2}^{\mathrm{a}}\right)\right] u_{(12)}+\frac{1}{\Omega}\left[K_{1}\left(K_{1} \pm k_{1}\right)\right. \\
& \left.-K_{2}\left(K_{2} \pm k_{2}^{\mathrm{a}}\right)\right]\left(u_{11}^{\mathrm{a}}-u_{22}^{\mathrm{a}}\right)+\mu_{0}\left[\left(\varepsilon_{0}+\mathrm{P}_{3311}^{I I}\right) u_{11}^{\mathrm{a}}+\left(\varepsilon_{0}+\mathrm{P}_{3322}^{I I}\right) u_{22}^{\mathrm{a}}\right. \\
& \left.\left.+\left(-\varepsilon_{0}+\mathrm{P}_{3333}^{I I}\right) u_{33}^{\mathrm{a}}\right](\Omega \pm \omega)\right\}(\Omega \pm \omega) \frac{E_{3}}{2} \\
\Phi_{ \pm}^{\mathrm{a}}= & \left(K_{1} \pm k_{1}\right) \mathrm{X}_{1}+\left(K_{2} \pm k_{2}^{\mathrm{a}}\right) \mathrm{X}_{2}-(\Omega \pm \omega) \mathrm{t}+\left(\Phi_{0} \pm \varphi_{0}\right) \\
\Phi_{ \pm}^{\mathrm{Ra}}= & \left(K_{1} \pm k_{1}\right) \mathrm{X}_{1}-\left(K_{2} \mp k_{2}^{\mathrm{a}}\right) \mathrm{X}_{2}-(\Omega \pm \omega) \mathrm{t}+\left(\Phi_{0} \pm \varphi_{0}\right)
\end{aligned}
$$

with

$$
u_{\mathrm{AB}}^{\mathrm{a}}=k_{\mathrm{B}}^{\mathrm{a}} u_{\mathrm{A}}
$$

The term $\Lambda^{\mathrm{Ra}}$ is obtained from $\Lambda^{\mathrm{a}}$ by replacing $K_{2}$ with $-K_{2}$. In the derivation of (53), the relations $k_{1}^{\mathrm{a}}=k_{1}(\mathrm{a}=\mathrm{I} \sim \mathrm{IV})$ which shown in Table 2 has been used.

The perturbed electromagnetic fields within the dielectric slab waveguide $\left|\mathrm{X}_{2}\right|<\frac{\mathrm{d}}{2}$ can be obtained from (47) and (53) together with (54) as

$$
\mathrm{e}_{3}^{-}=\sum_{+,-} \Re e \mathrm{e}_{3 \pm}^{\mathrm{S}}, \quad \mathrm{b}_{1}^{-}=\sum_{+,-} \Re e \mathrm{~b}_{1 \pm}^{\mathrm{S}}, \quad \mathrm{b}_{2}^{-}=\sum_{+,-} \Re e \mathrm{~b}_{2 \pm}^{\mathrm{S}}
$$


where

$$
\begin{aligned}
\mathrm{e}_{3 \pm}^{\mathrm{S}}= & \sum_{\mathrm{a}=\mathrm{I}}^{\mathrm{IV}}\left[e_{3 \pm}^{\mathrm{a}} \exp \left(i \Phi_{ \pm}^{\mathrm{a}}\right)+e_{3 \pm}^{\mathrm{Ra}} \exp \left(i \Phi_{ \pm}^{\mathrm{Ra}}\right)\right] \\
& +e_{3 \pm}^{\mathrm{U}} \exp \left(i \Phi_{ \pm}^{\mathrm{U}}\right)+e_{3 \pm}^{\mathrm{D}} \exp \left(i \Phi_{ \pm}^{\mathrm{D}}\right) \\
\mathrm{b}^{\mathrm{S}}{ }_{1 \pm}= & \frac{-i}{\Omega \pm \omega} \frac{\partial \mathrm{e}^{\mathrm{S}}{ }_{3 \pm}}{\partial \mathrm{X}_{2}}, \quad \mathrm{~b}^{\mathrm{S}}{ }_{2 \pm}=-\frac{K_{1} \pm k_{1}}{\Omega \pm \omega} \mathrm{e}^{\mathrm{S}}{ }_{3 \pm}
\end{aligned}
$$

with

$$
\begin{aligned}
e_{3 \pm}^{\mathrm{a}} & =\frac{\Lambda_{3 \pm}^{\mathrm{a}}}{\mathrm{D}_{ \pm}^{\mathrm{a}}}, \quad e_{3 \pm}^{\mathrm{Ra}}=\frac{\Lambda_{3 \pm}^{\mathrm{Ra}}}{\mathrm{D}_{ \pm}^{\mathrm{Ra}}} \\
\mathrm{D}_{ \pm}^{\mathrm{a}} & =\left(K_{1} \pm k_{1}\right)^{2}+\left(K_{2} \pm k_{2}^{\mathrm{a}}\right)^{2}-\mu_{0} \varepsilon_{33}(\Omega \pm \omega)^{2} \\
\mathrm{D}_{ \pm}^{\mathrm{Ra}} & =\left(K_{1} \pm k_{1}\right)^{2}+\left(-K_{2} \pm k_{2}^{\mathrm{a}}\right)^{2}-\mu_{0} \varepsilon_{33}(\Omega \pm \omega)^{2} \\
\Phi_{ \pm}^{\mathrm{U}} & =\left(K_{1} \pm k_{1}\right) \mathrm{X}_{1}+K_{2 \pm}^{\mathrm{h}} \mathrm{X}_{2}-(\Omega \pm \omega) \mathrm{t}+\left(\Phi_{ \pm}^{\mathrm{U} 0} \pm \varphi_{0}\right) \\
\Phi_{ \pm}^{\mathrm{D}} & =\left(K_{1} \pm k_{1}\right) \mathrm{X}_{1}-K_{2 \pm}^{\mathrm{h}} \mathrm{X}_{2}-(\Omega \pm \omega) \mathrm{t}+\left(\Phi_{ \pm}^{\mathrm{D} 0} \pm \varphi_{0}\right) \\
K_{2 \pm}^{\mathrm{h}} & =\left[\mu_{0} \varepsilon_{33}(\Omega \pm \omega)^{2}-\left(K_{1} \pm k_{1}\right)^{2}\right]^{\frac{1}{2}}
\end{aligned}
$$

The homogeneous solutions of (53) with the superscript $U$ and D stand for the quantities propagating upward and downward, respectively. Once the denominator $\mathrm{D}_{ \pm}^{\mathrm{a}}$ or $\mathrm{D}_{ \pm}^{\mathrm{Ra}}$ equals to zero, the phase matching condition is satisfied $[13,15]$. For such a case, the particular solution becomes the same form as homogeneous solution, then it is necessary to take higher order term solution which grows linearly along the propagating direction [14]. Nevertheless, the particular solution will decay rapidly since it violates all the characteristic equations of guided mode of electromagnetic wave.

Alternatively, the perturbed electromagnetic fields in the vacuum side can be expressed in two regions: 1. $X_{2}>\frac{d}{2}$

$$
\begin{aligned}
& \left.\mathrm{e}_{3}^{+}=\sum_{+,-} \Re e\left\{e_{A \pm}^{\mathrm{H}} \exp \left[-\alpha_{ \pm}\left(\mathrm{X}_{2}-\frac{\mathrm{d}}{2}\right)+i \Phi_{ \pm}^{\mathrm{H}}\right)\right]\right\} \\
& \mathrm{b}_{\mathrm{B}}^{+}=\sum_{+,-} \Re e\left\{b_{\mathrm{B} \pm}^{\mathrm{H}} \exp \left[-\alpha_{ \pm}\left(\mathrm{X}_{2}-\frac{\mathrm{d}}{2}\right)+i\left(\Phi_{ \pm}^{\mathrm{H}}+\Phi_{\mathrm{B}}\right)\right]\right\} \quad \mathrm{B}=1,2
\end{aligned}
$$

where

$$
\begin{aligned}
b_{1 \pm}^{\mathrm{H}} & =\frac{-i \alpha_{ \pm}}{\left(K_{1} \pm k_{1}\right)} b_{2 \pm}^{\mathrm{H}}=\frac{i \alpha_{ \pm}}{(\Omega \pm \omega)} e_{3 \pm}^{\mathrm{H}} \\
\alpha_{ \pm} & =\left[\left(K_{1} \pm k_{1}\right)^{2}-\mu_{0} \varepsilon_{0}(\Omega \pm \omega)^{2}\right]^{\frac{1}{2}} \\
\Phi_{ \pm}^{\mathrm{H}} & =\left(K_{1} \pm k_{1}\right) \mathrm{X}_{1}-(\Omega \pm \omega) \mathrm{t}+\Phi^{\mathrm{H} 0} \pm \varphi_{0} \\
\Phi_{1} & =-\frac{\pi}{2}, \quad \Phi_{2}=0
\end{aligned}
$$


68 C.-S. Yeh and C.-B. Lin / Mode transition of electromagnetic wave in slab waveguide subjected to in-plane elastic wave

2. $\mathrm{X}_{2}<-\frac{\mathrm{d}}{2}$

$$
\begin{aligned}
& \left.\mathrm{e}_{3}^{+}=\sum_{+,-} \Re e\left\{e_{A \pm}^{\mathrm{H}} \exp \left[\alpha_{ \pm}\left(\mathrm{X}_{2}+\frac{\mathrm{d}}{2}\right)+i \Phi_{ \pm}^{\mathrm{L}}\right)\right]\right\} \\
& \mathrm{b}_{\mathrm{B}}^{+}=\sum_{+,-} \Re e\left\{b_{\mathrm{B} \pm}^{\mathrm{L}} \exp \left[\alpha_{ \pm}\left(\mathrm{X}_{2}+\frac{\mathrm{d}}{2}\right)+i\left(\Phi_{ \pm}^{\mathrm{L}}+\Phi_{\mathrm{B}}\right)\right]\right\} \quad \mathrm{B}=1,2
\end{aligned}
$$

where

$$
b_{1 \pm}^{\mathrm{L}}=\frac{i \alpha_{ \pm}}{\left(K_{1} \pm k_{1}\right)} b_{2 \pm}^{\mathrm{L}}=\frac{-i \alpha_{ \pm}}{(\Omega \pm \omega)} e_{3 \pm}^{\mathrm{L}}
$$

The terms $\alpha_{ \pm}, \Phi_{ \pm}^{\mathrm{H}}$ and $\Phi_{1,2}$ are defined in the same form as those shown in (60) with the superscripts $\mathrm{L}$ instead of $\mathrm{H}$.

From (43), (45) and Table 1, the boundary conditions of the perturbed fields can be expressed for the present case as

$$
\begin{aligned}
\mathrm{e}_{3}^{-}=\mathrm{e}_{3}^{+}+\left(i K_{1} \mathrm{u}_{1}^{-}-\alpha \mathrm{u}_{2}^{-}\right) \mathrm{E}_{3}^{0+} & \\
\mathrm{b}_{2}^{-}=\mathrm{b}_{2}^{+}+\left(i K_{1} \mathrm{u}_{1}^{-}-\alpha \mathrm{u}_{2}^{-}\right) \mathrm{B}_{2}^{0+}+\left[\mathrm{u}_{C, C}^{-} \mathrm{B}_{2}^{0+}-\right. & \left.\left(\mathrm{u}_{2,1}^{-} \mathrm{B}_{1}^{0+}+\mathrm{u}_{2,2}^{-} \mathrm{B}_{2}^{0+}\right)\right] \\
\mathrm{b}_{1}^{-}+\left(2 \mathrm{u}_{1,1}^{-}-\mathrm{u}_{E, E}^{-}\right) \mathcal{B}_{1}^{0-}+\left(\mathrm{u}_{1,2}^{-}+\mathrm{u}_{2,1}^{-}\right) \mathcal{B}_{2}^{0-}= & \mathrm{b}_{1}^{+}+\left(i K_{1} \mathrm{u}_{1}^{-}-\alpha \mathrm{u}_{2}^{-}\right) \mathrm{B}_{1}^{0+} \\
& +\mathrm{u}_{1,1}^{-} \mathrm{B}_{1}^{0+}+\mathrm{u}_{2,1}^{-} \mathrm{B}_{2}^{0+}
\end{aligned}
$$

On the basis of the following estimations

$$
\begin{aligned}
& \frac{K_{1} \pm k_{1}}{\Omega \pm \omega}=\frac{K_{1}}{\Omega}\left(1 \pm \frac{k_{1}}{K_{1}} \mp \frac{\omega}{\Omega}+\text { H.O.T. }\right) \\
& \frac{k_{1} / K_{1}}{\omega / \Omega}=\frac{k_{1} / \omega}{K_{1} / \Omega} \sim\left(\frac{c_{L}}{c}\right) \ll 1
\end{aligned}
$$

we can obtain

$$
\frac{K}{\Omega} \cdot \mathrm{e}_{3}^{-}+\mathrm{b}_{2}^{-} \sim O\left(\frac{k_{1}}{K_{1}}\right)
$$

Note that in the derivations of (65), the terms with order of magnitude $\frac{k_{1}}{K_{1}}$ has been neglected [14], and (63b) can be obtained from (63a).

The substitutions of (56), (59) and (61) into the boundary conditions (63 a, c) will lead to sixteen independent equations to determine sixteen unknowns $e_{3 \pm}^{\mathrm{U}}, \Phi_{ \pm}^{\mathrm{U} 0}, e_{3 \pm}^{\mathrm{D}}, \Phi_{ \pm}^{\mathrm{D} 0}, e_{3 \pm}^{\mathrm{H}}, \Phi_{ \pm}^{\mathrm{H} 0}, e_{3 \pm}^{\mathrm{L}}$ and $\Phi_{ \pm}^{\mathrm{L} 0}$. A unique solution may be found for such a case. However, since the features of electromagnetic fields in dielectric slab rather than the vacuum side is relavant in this study, only those unknown quantities appearred in (57) and (58) are listed below. Furthermore, in order to investigate the character of the perturbed field, the solution of $e_{3 \pm}^{\mathrm{U}}, \Phi_{ \pm}^{\mathrm{U} 0}, e_{3 \pm}^{\mathrm{D}}$ and $\Phi_{ \pm}^{\mathrm{D} 0}$ can be rearranged as follows:

$$
\mathrm{q}_{ \pm}=\frac{\mathrm{Q}_{ \pm}}{\mathrm{U}_{ \pm}}, \quad \mathrm{r}_{ \pm}=\frac{\mathrm{R}_{ \pm}}{\mathrm{V}_{ \pm}}, \quad \mathrm{s}_{ \pm}=\frac{\mathrm{S}_{ \pm}}{\mathrm{U}_{ \pm}}, \quad \mathrm{w}_{ \pm}=\frac{\mathrm{W}_{ \pm}}{\mathrm{V}_{ \pm}}
$$


where

$$
\begin{aligned}
& \mathrm{q}_{ \pm}=e_{3 \pm}^{\mathrm{U}} \cos \left(\Phi_{ \pm}^{\mathrm{U} 0}-\Phi_{0}\right)+e_{3 \pm}^{\mathrm{D}} \cos \left(\Phi_{ \pm}^{\mathrm{D} 0}-\Phi_{0}\right) \\
& \mathrm{r}_{ \pm}=e_{3 \pm}^{\mathrm{U}} \cos \left(\Phi_{ \pm}^{\mathrm{U} 0}-\Phi_{0}\right)-e_{3 \pm}^{\mathrm{D}} \cos \left(\Phi_{ \pm}^{\mathrm{D} 0}-\Phi_{0}\right) \\
& \mathrm{s}_{ \pm}=e_{3 \pm}^{\mathrm{U}} \sin \left(\Phi_{ \pm}^{\mathrm{U} 0}-\Phi_{0}\right)+e_{3 \pm}^{\mathrm{D}} \sin \left(\Phi_{ \pm}^{\mathrm{D} 0}-\Phi_{0}\right) \\
& \mathrm{w}_{ \pm}=e_{3 \pm}^{\mathrm{U}} \sin \left(\Phi_{ \pm}^{\mathrm{U} 0}-\Phi_{0}\right)-e_{3 \pm}^{\mathrm{D}} \sin \left(\Phi_{ \pm}^{\mathrm{D} 0}-\Phi_{0}\right)
\end{aligned}
$$

with

$$
\begin{aligned}
& \mathrm{Q}_{ \pm}=\sum_{\mathrm{a}=\mathrm{III}}^{\mathrm{IV}}\left(\mathrm{Q}_{ \pm}^{\mathrm{ac}} \mathrm{C}_{2 \pm}^{\mathrm{ad}}+\mathrm{Q}_{ \pm}^{\mathrm{as}} \mathrm{S}_{2 \pm}^{\mathrm{ad}}\right), \quad \mathrm{R}_{ \pm}=\sum_{\mathrm{a}=\mathrm{III}}^{\mathrm{IV}}\left(\mathrm{R}_{ \pm}^{\mathrm{ac}} \mathrm{C}_{2 \pm}^{\mathrm{ad}}+\mathrm{R}_{ \pm}^{\mathrm{as}} \mathrm{S}_{2 \pm}^{\mathrm{ad}}\right) \\
& \mathrm{S}_{ \pm}=\sum_{\mathrm{a}=\mathrm{I}}^{\mathrm{II}}\left(\mathrm{S}_{ \pm}^{\mathrm{ac}} \mathrm{C}_{2 \pm}^{\mathrm{ad}}+\mathrm{S}_{ \pm}^{\mathrm{as}} \mathrm{S}_{2 \pm}^{\mathrm{ad}}\right), \quad \mathrm{W}_{ \pm}=\sum_{\mathrm{a}=\mathrm{I}}^{\mathrm{II}}\left(\mathrm{T}_{ \pm}^{\mathrm{ac}} \mathrm{C}_{2 \pm}^{\mathrm{ad}}+\mathrm{T}_{ \pm}^{\mathrm{as}} \mathrm{S}_{2 \pm}^{\mathrm{ad}}\right) \\
& \mathrm{U}_{ \pm}=\alpha_{ \pm} \cos \frac{K_{2 \pm}^{\mathrm{h}} \mathrm{d}}{2}-K_{2 \pm}^{\mathrm{h}} \sin \frac{K_{2 \pm}^{\mathrm{h}} \mathrm{d}}{2}, \quad \mathrm{~V}_{ \pm}=\alpha_{ \pm} \sin \frac{K_{2 \pm}^{\mathrm{h}} \mathrm{d}}{2}+K_{2 \pm}^{\mathrm{h}} \cos \frac{K_{2 \pm}^{\mathrm{h}} \mathrm{d}}{2}
\end{aligned}
$$

and

$$
\begin{aligned}
\mathrm{Q}_{ \pm}^{\mathrm{ac}} & =-\alpha_{ \pm}\left(e_{3 \pm}^{\mathrm{a}}+e_{3 \pm}^{\mathrm{Rb}}\right)+e_{ \pm}^{\mathrm{ac}}, \quad \mathrm{Q}_{ \pm}^{\mathrm{as}}=\left[\left(K_{2} \pm k_{2}^{\mathrm{a}}\right) e_{3 \pm}^{\mathrm{a}}+\left(K_{2} \pm k_{2}^{\mathrm{b}}\right) e_{3 \pm}^{\mathrm{Rb}}\right]-e_{ \pm}^{\mathrm{as}} \\
\mathrm{R}_{ \pm}^{\mathrm{ac}} & =-\left[\left(K_{2} \pm k_{2}^{\mathrm{a}}\right) e_{3 \pm}^{\mathrm{a}}-\left(K_{2} \pm k_{2}^{\mathrm{b}}\right) e_{3 \pm}^{\mathrm{Rb}}\right]+e_{ \pm}^{\mathrm{as}}, \quad \mathrm{R}_{ \pm}^{\mathrm{as}}=-\alpha_{ \pm}\left(e_{3 \pm}^{\mathrm{a}}-e_{3 \pm}^{\mathrm{Rb}}\right)+e_{ \pm}^{\mathrm{ac}} \\
\mathrm{S}_{ \pm}^{\mathrm{ac}} & = \pm \alpha_{ \pm}\left(e_{3 \pm}^{\mathrm{a}}-e_{3 \pm}^{\mathrm{Rb}}\right)-e_{ \pm}^{\mathrm{ac}}, \quad \mathrm{S}_{ \pm}^{\mathrm{as}}=\mp\left[\left(K_{2} \pm k_{2}^{\mathrm{a}}\right) e_{3 \pm}^{\mathrm{a}}-\left(K_{2} \pm k_{2}^{\mathrm{b}}\right) e_{3 \pm}^{\mathrm{Rb}}\right]-e_{ \pm}^{\mathrm{as}} \\
\mathrm{W}_{ \pm}^{\mathrm{ac}} & = \pm\left[\left(K_{2} \pm k_{2}^{\mathrm{a}}\right) e_{3 \pm}^{\mathrm{a}}+\left(K_{2} \pm k_{2}^{\mathrm{b}}\right) e_{3 \pm}^{\mathrm{Rb}}\right]-e_{ \pm}^{\mathrm{as}}, \quad \mathrm{W}_{ \pm}^{\mathrm{as}}= \pm \alpha_{ \pm}\left(e_{3 \pm}^{\mathrm{a}}+e_{3 \pm}^{\mathrm{Rb}}\right)-e_{ \pm}^{\mathrm{ac}} \\
\mathrm{C}_{2 \pm}^{\mathrm{ad}} & =\cos \frac{\left(K_{2} \pm k_{2}^{\mathrm{a}}\right) \mathrm{d}}{2}, \quad \mathrm{~S}_{2 \pm}^{\mathrm{ad}}=\sin \frac{\left(K_{2} \pm k_{2}^{\mathrm{a}}\right) \mathrm{d}}{2} \\
e_{ \pm}^{\mathrm{ac}} & =\frac{E_{3}}{2}\left[ \pm\left(\alpha_{ \pm}-\alpha\right) K_{1} u_{1}^{\mathrm{a}}-\alpha u_{22}^{\mathrm{a}}\right], \quad e_{ \pm}^{\mathrm{as}}=\frac{E_{3}}{2}\left[ \pm\left(\alpha_{ \pm}-\alpha\right) \alpha u_{2}^{\mathrm{a}}-K_{1} u_{12}^{\mathrm{a}}\right]
\end{aligned}
$$

The superscript a denotes I, II, III or IV, and the corresponding b is II, I, IV or III sequentially. The features of (66) can be presented in Table 3 for various conditions.

In order to interpret the character of mode transition of guided electromagnetic wave as shown in the first four column of Table 3, the real part of (57) can be rearranged as follows:

$$
\begin{aligned}
& \Re \mathrm{e}\left(e_{3 \pm}^{\mathrm{S}}\right)=e_{3 \pm}^{\mathrm{SC}} \mathrm{C}_{1 \pm}+e_{3 \pm}^{\mathrm{SS}} \mathrm{S}_{1 \pm} \\
& \Re \mathrm{e}\left(b_{\mathrm{A} \pm}^{\mathrm{S}}\right)=b_{\mathrm{A} \pm}^{\mathrm{SC}} \mathrm{C}_{1 \pm}+b_{\mathrm{A} \pm}^{\mathrm{SS}} \mathrm{S}_{1 \pm} \quad \mathrm{A}=1,2
\end{aligned}
$$

where

$$
e_{3 \pm}^{\mathrm{SC}}=\left[\sum_{\mathrm{a}=\mathrm{I}}^{\mathrm{IV}}\left(e_{3 \pm}^{\mathrm{a}}+e_{3 \pm}^{\mathrm{Rb}}\right) \mathrm{C}_{2 \pm}^{\mathrm{a}}\right]+\mathrm{q}_{ \pm} \mathrm{C}_{2 \pm}^{\mathrm{h}}-\mathrm{t}_{ \pm} \mathrm{S}_{2 \pm}^{\mathrm{h}}
$$


70 C.-S. Yeh and C.-B. Lin / Mode transition of electromagnetic wave in slab waveguide subjected to in-plane elastic wave

Table 3

The mode transition of guided electromagnetic wave

\begin{tabular}{cccccc}
\hline case no. & $\begin{array}{c}\mathrm{I} . \\
\left(\mathrm{U}_{+}=0\right)\end{array}$ & $\begin{array}{c}\mathrm{II} . \\
\left(\mathrm{U}_{-}=0\right)\end{array}$ & $\begin{array}{c}\mathrm{III} . \\
\left(\mathrm{V}_{+}=0\right)\end{array}$ & $\begin{array}{c}\mathrm{IV} . \\
\left(\mathrm{V}_{-}=0\right)\end{array}$ & $\begin{array}{c}\mathrm{V} . \\
\left(\mathrm{U}_{ \pm}, \mathrm{V}_{ \pm} \neq 0\right)\end{array}$ \\
\hline $\mathrm{q}_{+}$ & $\infty$ & $\mathrm{Q}_{+} / \mathrm{U}_{+}$ & $\mathrm{Q}_{+} / \mathrm{U}_{+}$ & $\mathrm{Q}_{+} / \mathrm{U}_{+}$ & $\mathrm{Q}_{+} / \mathrm{U}_{+}$ \\
$\mathrm{q}_{-}$ & $\mathrm{Q}_{-} / \mathrm{U}_{-}$ & $\infty$ & $\mathrm{Q}_{-} / \mathrm{U}_{-}$ & $\mathrm{Q}_{-} / \mathrm{U}_{-}$ & $\mathrm{Q}_{-} / \mathrm{U}_{-}$ \\
$\mathrm{r}_{+}$ & $\mathrm{R}_{+} / \mathrm{V}_{+}$ & $\mathrm{R}_{+} / \mathrm{V}_{+}$ & $\infty$ & $\mathrm{R}_{+} / \mathrm{V}_{+}$ & $\mathrm{R}_{+} / \mathrm{V}_{+}$ \\
$\mathrm{r}_{-}$ & $\mathrm{R}_{-} / \mathrm{V}_{-}$ & $\mathrm{R}_{-} / \mathrm{V}_{-}$ & $\mathrm{R}_{-} / \mathrm{V}_{-}$ & $\infty$ & $\mathrm{R}_{-} / \mathrm{V}_{-}$ \\
$\mathrm{S}_{+}$ & $\infty$ & $\mathrm{S}_{+} / \mathrm{U}_{+}$ & $\mathrm{S}_{+} / \mathrm{U}_{+}$ & $\mathrm{S}_{+} / \mathrm{U}_{+}$ & $\mathrm{S}_{+} / \mathrm{U}_{+}$ \\
$\mathrm{S}_{-}$ & $\mathrm{S}_{-} / \mathrm{U}_{-}$ & $\infty$ & $\mathrm{S}_{-} / \mathrm{U}_{-}$ & $\mathrm{S}_{-} / \mathrm{U}_{-}$ & $\mathrm{S}_{-} / \mathrm{U}_{-}$ \\
$\mathrm{w}_{+}$ & $\mathrm{W}_{+} / \mathrm{V}_{+}$ & $\mathrm{W}_{+} / \mathrm{V}_{+}$ & $\infty$ & $\mathrm{W}_{+} / \mathrm{V}_{+}$ & $\mathrm{W}_{+} / \mathrm{V}_{+}$ \\
$\mathrm{w}_{-}$ & $\mathrm{W}_{-} / \mathrm{V}_{-}$ & $\mathrm{W}_{-} / \mathrm{V}_{-}$ & $\mathrm{W}_{-} / \mathrm{V}_{-}$ & $\infty$ & $\mathrm{W}_{-} / \mathrm{V}_{-}$ \\
new mode & odd TE & odd TE & even TE & even TE & none \\
\hline
\end{tabular}

$$
\begin{aligned}
e_{3 \pm}^{\mathrm{SS}} & =\left[\sum_{\mathrm{a}=\mathrm{I}}^{\mathrm{IV}}\left(-e_{3 \pm}^{\mathrm{a}}+e_{3 \pm}^{\mathrm{Rb}}\right) \mathrm{S}_{2 \pm}^{\mathrm{a}}\right]-\mathrm{S}_{ \pm} \mathrm{C}_{2 \pm}^{\mathrm{h}}-\mathrm{r}_{ \pm} \mathrm{S}_{2 \pm}^{\mathrm{h}} \\
b_{1 \pm}^{\mathrm{SC}} & =\frac{1}{\Omega \pm \omega}\left\{\sum_{\mathrm{a}=\mathrm{I}}^{\mathrm{IV}}\left[\left(K_{2} \pm k_{2}^{\mathrm{a}}\right) e_{3 \pm}^{\mathrm{a}}-\left(K_{2} \pm k_{2}^{\mathrm{b}}\right) e_{3 \pm}^{\mathrm{Rb}}\right] \mathrm{C}_{2 \pm}^{\mathrm{a}}+K_{2 \pm}^{\mathrm{h}}\left(\mathrm{r}_{ \pm} \mathrm{C}_{2 \pm}^{\mathrm{h}}-\mathrm{s}_{ \pm} \mathrm{S}_{2 \pm}^{\mathrm{h}}\right)\right\} \\
b_{1 \pm}^{\mathrm{SS}} & =\frac{-1}{\Omega \pm \omega}\left\{\sum_{\mathrm{a}=\mathrm{I}}^{\mathrm{IV}}\left[\left(K_{2} \pm k_{2}^{\mathrm{a}}\right) e_{3 \pm}^{\mathrm{a}}+\left(K_{2} \pm k_{2}^{\mathrm{b}}\right) e_{3 \pm}^{\mathrm{Rb}}\right] \mathrm{S}_{2 \pm}^{\mathrm{a}}+K_{2 \pm}^{\mathrm{h}}\left(\mathrm{t}_{ \pm} \mathrm{C}_{2 \pm}^{\mathrm{h}}+\mathrm{q}_{ \pm} \mathrm{S}_{2 \pm}^{\mathrm{h}}\right)\right\} \\
b_{2 \pm}^{\mathrm{SC}} & =-\frac{K_{1} \pm k_{1}}{\Omega \pm \omega} e_{3 \pm}^{\mathrm{SC}}, \quad b_{2 \pm}^{\mathrm{SS}}=-\frac{K_{1} \pm k_{1}}{\Omega \pm \omega} e_{3 \pm}^{\mathrm{SS}} \\
\mathrm{C}_{1 \pm} & =\cos \left[\left(K_{1} \pm k_{1}\right) \mathrm{X}_{1}-(\Omega \pm \omega)+\Phi_{0} \pm \varphi_{0}\right] \\
\mathrm{S}_{1 \pm} & =\sin \left[\left(K_{1} \pm k_{1}\right) \mathrm{X}_{1}-(\Omega \pm \omega)+\Phi_{0} \pm \varphi_{0}\right]
\end{aligned}
$$

with

$$
\begin{aligned}
& \mathrm{C}_{2 \pm}^{\mathrm{a}}=\cos \left[\left(K_{2} \pm k_{2}^{\mathrm{a}}\right) \mathrm{X}_{2} \pm\left(\varphi_{0}^{\mathrm{a}}-\varphi_{0}\right)\right], \quad \mathrm{S}_{2 \pm}^{\mathrm{a}}=\sin \left[\left(K_{2} \pm k_{2}^{\mathrm{a}}\right) \mathrm{X}_{2} \pm\left(\varphi_{0}^{\mathrm{a}}-\varphi_{0}\right)\right] \\
& \mathrm{C}_{2 \pm}^{\mathrm{h}}=\cos \left(K_{2 \pm}^{\mathrm{h}} \mathrm{X}_{2}\right), \quad \mathrm{S}_{2 \pm}^{\mathrm{h}}=\sin \left(K_{2 \pm}^{\mathrm{h}} \mathrm{X}_{2}\right)
\end{aligned}
$$

Note that the superscripts $\mathrm{a}$ and $\mathrm{b}$ are in the same corresponding pair as in (68). As shown in Table 1, whether the mode is odd or even depends on the appearrance of $\mathrm{C}_{2}$ or $\mathrm{S}_{2}$, and $\mathrm{q}_{ \pm}, \mathrm{r}_{ \pm}$and $\mathrm{s}_{ \pm}, \mathrm{w}_{ \pm}$are the corresponding coefficients of odd and even TE modes, respectively. Furthermore, since conditions for the first two column of Table 1 satisfy the characteristic equation of odd TE mode, the physical meaning of $\mathrm{q}_{ \pm}$(or $\mathrm{r}_{ \pm}$) approaching infinity is that the electromagnetic energy transmits from the original odd TE mode to another odd TE mode under the action of certain perturbed elastic wave. Of course, the amplitude of the new guided mode can not exceed the original one.

The last column in Table 3 does not concern with the mode transition of electromagnetic wave. For such a situation, the coefficients in perturbed wave can be rearranged as

$$
\begin{aligned}
& e_{3 \pm}^{\mathrm{U}}=\frac{1}{2}\left[\left(\frac{\mathrm{Q}_{ \pm}}{\mathrm{U}_{ \pm}}+\frac{\mathrm{R}_{ \pm}}{\mathrm{V}_{ \pm}}\right)^{2}+\left(\frac{\mathrm{S}_{ \pm}}{\mathrm{U}_{ \pm}}+\frac{\mathrm{W}_{ \pm}}{\mathrm{V}_{ \pm}}\right)^{2}\right]^{\frac{1}{2}} \\
& e_{3 \pm}^{\mathrm{D}}=\frac{1}{2}\left[\left(\frac{\mathrm{Q}_{ \pm}}{\mathrm{U}_{ \pm}}-\frac{\mathrm{R}_{ \pm}}{\mathrm{V}_{ \pm}}\right)^{2}+\left(\frac{\mathrm{S}_{ \pm}}{\mathrm{U}_{ \pm}}-\frac{\mathrm{W}_{ \pm}}{\mathrm{V}_{ \pm}}\right)^{2}\right]^{\frac{1}{2}}
\end{aligned}
$$


C.-S. Yeh and C.-B. Lin / Mode transition of electromagnetic wave in slab waveguide subjected to in-plane elastic wave 71

$$
\begin{aligned}
& \Phi_{ \pm}^{\mathrm{U} 0}=\Phi_{0}-\tan ^{-1} \frac{\mathrm{S}_{ \pm} \mathrm{V}_{ \pm}+\mathrm{W}_{ \pm} \mathrm{U}_{ \pm}}{\mathrm{Q}_{ \pm} \mathrm{V}_{ \pm}+\mathrm{R}_{ \pm} \mathrm{U}_{ \pm}} \\
& \Phi_{ \pm}^{\mathrm{D} 0}=\Phi_{0}-\tan ^{-1} \frac{S_{ \pm} \mathrm{V}_{ \pm}-\mathrm{W}_{ \pm} \mathrm{U}_{ \pm}}{\mathrm{Q}_{ \pm} \mathrm{V}_{ \pm}-\mathrm{R}_{ \pm} \mathrm{U}_{ \pm}}
\end{aligned}
$$

Although the perturbed electromagnetic wave is expressed in a standing wave form along $\mathrm{X}_{2}$-direction just like the original one, it propagates along $\mathrm{X}_{1}$-direction. Therefore, the total electric field can be rearranged as

$$
\mathrm{E}_{3}^{-}=\mathcal{E}_{3}^{0-}+\mathrm{e}_{3}^{-}=\Re \mathrm{e}\left[E_{3}^{\mathrm{S}} \exp \left(i \Phi^{\mathrm{S}}\right)\right]
$$

where

$$
E_{3}^{\mathrm{S}}=\left[\left(E_{3 \mathrm{C}}^{\mathrm{S}}\right)^{2}+\left(E_{3 \mathrm{~S}}^{\mathrm{S}}\right)^{2}\right]^{\frac{1}{2}}, \quad \Phi^{\mathrm{S}}=\tan ^{-1} \frac{E_{3 \mathrm{~S}}^{\mathrm{S}}}{E_{3 \mathrm{C}}^{\mathrm{S}}} \simeq \Phi^{\mathrm{m}}+\Theta^{\mathrm{m}}
$$

with

$$
\begin{aligned}
E_{3 \mathrm{C}}^{\mathrm{S}} & =E_{3}^{*} \cos (\Phi+\Theta)+E_{3}^{\mathrm{R} *} \cos \left(\Phi^{\mathrm{R}}+\Theta^{\mathrm{R}}\right) \\
& \simeq\left(E_{3}^{*}+E_{3}^{\mathrm{R} *}\right) \cos \left(\Phi^{\mathrm{m}}+\Theta^{\mathrm{m}}\right) \cos \left(\Phi^{\mathrm{d}}+\Theta^{\mathrm{d}}\right) \\
E_{3 \mathrm{~S}}^{\mathrm{S}} & =E_{3}^{*} \sin (\Phi+\Theta)+E_{3}^{\mathrm{R} *} \sin \left(\Phi^{\mathrm{R}}+\Theta^{\mathrm{R}}\right) \\
& \simeq\left(E_{3}^{*}+E_{3}^{\mathrm{R} *}\right) \sin \left(\Phi^{\mathrm{m}}+\Theta^{\mathrm{m}}\right) \cos \left(\Phi^{\mathrm{d}}+\Theta^{\mathrm{d}}\right)
\end{aligned}
$$

Some parameters used in (76) should be defined as

$$
\begin{aligned}
E_{3}^{*} & =\left[\left(E_{3 C}\right)^{2}+\left(E_{3 S}\right)^{2}\right]^{\frac{1}{2}}, \quad E_{3}^{\mathrm{R} *}=\left[\left(E_{3 C}^{\mathrm{R}}\right)^{2}+\left(E_{3 S}^{\mathrm{R}}\right)^{2}\right]^{\frac{1}{2}} \\
\Phi & =K_{1} \mathrm{X}_{1}+K_{2} \mathrm{X}_{2}-\Omega \mathrm{t}+\Phi_{0}, \quad \Phi^{\mathrm{R}}=K_{1} \mathrm{X}_{1}-K_{2} \mathrm{X}_{2}-\Omega \mathrm{t}+\Phi_{0} \\
\Theta & =\tan ^{-1} \frac{E_{3 S}}{E_{3 C}}, \quad \Theta^{\mathrm{R}}=\tan ^{-1} \frac{E_{3 S}^{\mathrm{R}}}{E_{3 C}^{\mathrm{R}}}
\end{aligned}
$$

and

$$
\begin{aligned}
E_{3 C}= & E_{3}+\sum_{+,-}\left[\left(\sum_{\mathrm{a}=\mathrm{I}}^{\mathrm{IV}} e_{3 \pm}^{\mathrm{a}} \cos \eta^{\mathrm{a}}\right)+e_{3 \pm}^{\mathrm{U}} \cos \eta_{ \pm}^{\mathrm{U}}\right] \\
E_{3 S}= & \sum_{+,-} \pm\left[\left(\sum_{\mathrm{a}=\mathrm{I}}^{\mathrm{IV}} e_{3 \pm}^{\mathrm{a}} \sin \eta^{\mathrm{a}}\right)+e_{3 \pm}^{\mathrm{U}} \sin \eta_{ \pm}^{\mathrm{U}}\right] \\
E_{3 C}^{\mathrm{R}}= & E_{3}+\sum_{+,-}\left[\left(\sum_{\mathrm{a}=\mathrm{I}}^{\mathrm{IV}} e_{3 \pm}^{\mathrm{Ra}} \cos \eta^{\mathrm{a}}\right)+e_{3 \pm}^{\mathrm{D}} \cos \eta_{ \pm}^{\mathrm{D}}\right] \\
E_{3 S}^{\mathrm{R}}= & \sum_{+,-}\left[\left(\sum_{\mathrm{a}=\mathrm{I}}^{\mathrm{IV}} e_{3 \pm}^{\mathrm{Ra}} \sin \eta^{\mathrm{a}}\right)+e_{3 \pm}^{\mathrm{D}} \sin \eta_{ \pm}^{\mathrm{D}}\right] \\
\eta^{\mathrm{a}}= & k_{1} \mathrm{X}_{1}+k_{2}^{\mathrm{a}} \mathrm{X}_{2}-\omega \mathrm{t}+\varphi_{0} \\
\eta_{ \pm}^{\mathrm{D}}= & k_{1} \mathrm{X}_{1} \pm\left(K_{2}-K_{2 \pm}^{\mathrm{h}}\right) \mathrm{X}_{2}-\omega \mathrm{t}+\varphi_{0}+\left(\Phi_{ \pm}^{\mathrm{D} 0}-\Phi_{0}^{\mathrm{R}}\right) \\
\eta_{ \pm}^{\mathrm{U}}= & k_{1} \mathrm{X}_{1} \mp\left(K_{2}-K_{2 \pm}^{\mathrm{h}}\right) \mathrm{X}_{2}-\omega \mathrm{t}+\varphi_{0} \pm\left(\Phi_{ \pm}^{\mathrm{U} 0}-\Phi_{0}\right)
\end{aligned}
$$


72 C.-S. Yeh and C.-B. Lin / Mode transition of electromagnetic wave in slab waveguide subjected to in-plane elastic wave

Note that in the derivation of (76), the following relations has been used

$$
\begin{aligned}
& \Phi^{\mathrm{m}}=\frac{1}{2}\left(\Phi+\Phi^{\mathrm{R}}\right), \quad \Phi^{\mathrm{d}}=\frac{1}{2}\left(\Phi-\Phi^{\mathrm{R}}\right) \\
& \Theta^{\mathrm{m}}=\frac{1}{2}\left(\Theta+\Theta^{\mathrm{R}}\right), \quad \Theta^{\mathrm{d}}=\frac{1}{2}\left(\Theta-\Theta^{\mathrm{R}}\right) \\
& \frac{E_{3}^{*}-E_{3}^{\mathrm{R} *}}{E_{3}^{*}+E_{3}^{\mathrm{R} *}} \sim O\left(\frac{e_{3}^{\mathrm{a}}}{E_{3}}\right) \ll 1
\end{aligned}
$$

Making use of the estimation $O\left(\frac{k}{K}\right) \ll 1$ and the similar procedure in Ref.14, (68) can be approximated as

$$
\begin{aligned}
& \mathrm{Q}_{ \pm} \simeq \pm \sum_{\mathrm{a}=\mathrm{III}}^{\mathrm{IV}}\left(e_{3 \pm}^{\mathrm{a}}+e_{3 \pm}^{\mathrm{Rb}}\right) \mathrm{V} \sin \frac{k_{2}^{\mathrm{a}} \mathrm{d}}{2}, \quad \mathrm{R}_{ \pm} \simeq-\sum_{\mathrm{a}=\mathrm{III}}^{\mathrm{IV}}\left(e_{3 \pm}^{\mathrm{a}}-e_{3 \pm}^{\mathrm{Rb}}\right) \mathrm{V} \cos \frac{k_{2}^{\mathrm{a}} \mathrm{d}}{2} \\
& \mathrm{~S}_{ \pm} \simeq \sum_{\mathrm{a}=\mathrm{I}}^{\mathrm{II}}\left(e_{3 \pm}^{\mathrm{a}}-e_{3 \pm}^{\mathrm{Rb}}\right) \mathrm{V} \sin \frac{k_{2}^{\mathrm{a}} \mathrm{d}}{2}, \quad \mathrm{~W}_{ \pm} \simeq \pm \sum_{\mathrm{a}=\mathrm{I}}^{\mathrm{II}}\left(e_{3 \pm}^{\mathrm{a}}+e_{3 \pm}^{\mathrm{Rb}}\right) \mathrm{V} \cos \frac{k_{2}^{\mathrm{a}} \mathrm{d}}{2} \\
& \mathrm{U}_{ \pm}=\mathrm{V} \sin \frac{\left(K_{2}-k_{2 \pm}^{\mathrm{h}}\right) \mathrm{d}}{2}, \quad \mathrm{~V}_{ \pm}=\mathrm{V} \cos \frac{\left(K_{2}-k_{2 \pm}^{\mathrm{h}}\right) \mathrm{d}}{2}
\end{aligned}
$$

where

$$
\mathrm{V}=\alpha \sin \frac{K_{2} \mathrm{~d}}{2}+K_{2} \cos \frac{K_{2} \mathrm{~d}}{2}
$$

The characteristic equation of the original electromagnetic wave which is the lowest odd TE mode has been used in the derivation of (79). Thus, (73) can be approximated as

$$
\begin{array}{ll}
e_{3 \pm}^{\mathrm{U}} \simeq \frac{1}{\mathrm{~T}_{ \pm}}\left(\mathrm{Y}_{ \pm}^{2}+\mathrm{Z}_{ \pm}^{2}\right)^{\frac{1}{2}}, & e_{3 \pm}^{\mathrm{D}} \simeq \frac{1}{\mathrm{~T}_{ \pm}}\left(\mathrm{M}_{ \pm}^{2}+\mathrm{N}_{ \pm}^{2}\right)^{\frac{1}{2}} \\
\Phi_{ \pm}^{\mathrm{U} 0} \simeq \Phi_{0}-\tan ^{-1} \frac{\mathrm{Z}_{ \pm}^{2}}{\mathrm{Y}_{ \pm}^{2}}, \quad \Phi_{ \pm}^{\mathrm{D} 0} \simeq \Phi_{0}-\tan ^{-1} \frac{\mathrm{N}_{ \pm}^{2}}{\mathrm{M}_{ \pm}^{2}}
\end{array}
$$

where

$$
\begin{aligned}
& \mathrm{T}_{ \pm}=\sin \left[\left(K_{2}-K_{2 \pm}^{\mathrm{h}}\right) \mathrm{d}\right] \\
& \mathrm{Y}_{ \pm}=\sum_{\mathrm{a}=\mathrm{III}}^{\mathrm{IV}}\left(-e_{3 \pm}^{\mathrm{a}} \mathrm{S}_{ \pm}^{\mathrm{a}}+e_{3 \pm}^{\mathrm{Rb}} \mathrm{S}^{\mathrm{a} *}\right), \quad \mathrm{Z}_{ \pm}=\sum_{\mathrm{a}=\mathrm{I}}^{\mathrm{II}}\left( \pm e_{3 \pm}^{\mathrm{a}} \mathrm{S}^{\mathrm{a} *}{ }_{ \pm}+e_{3 \pm}^{\mathrm{Rb}} \mathrm{S}_{ \pm}^{\mathrm{a}}\right) \\
& \left.\mathrm{M}_{ \pm}=\sum_{\mathrm{a}=\mathrm{III}}^{\mathrm{IV}}\left(e_{3 \pm}^{\mathrm{a}} \mathrm{S}^{\mathrm{a} *}{ }_{ \pm}-e_{3 \pm}^{\mathrm{Rb}} \mathrm{S}_{ \pm}^{\mathrm{a}}\right), \quad \mathrm{N}_{ \pm}=\left[\sum_{\mathrm{a}=\mathrm{I}}^{\mathrm{II}}\left(\mp e_{3 \pm}^{\mathrm{a}} \mathrm{S}_{ \pm}^{\mathrm{a}}+e_{3 \pm}^{\mathrm{Rb} \mathrm{S}^{\mathrm{a} *}}{ }_{ \pm}\right)\right]\right\}^{\frac{1}{2}}
\end{aligned}
$$

with

$$
\mathrm{S}_{ \pm}^{\mathrm{a}}=\sin \frac{\left(K_{2}-K_{2 \pm}^{\mathrm{h}} \mp k_{2}^{\mathrm{a}}\right) \mathrm{d}}{2}, \quad \mathrm{~S}_{ \pm}^{\mathrm{a} *}=\sin \frac{\left(K_{2}-K_{2 \pm}^{\mathrm{h}} \pm k_{2}^{\mathrm{a}}\right) \mathrm{d}}{2} \quad \mathrm{a}=\mathrm{I} \sim \mathrm{IV}
$$


Making use of (78) and (82), the relative phase change of the total field can be written as

$$
\begin{aligned}
\left(\frac{\Delta \phi}{\phi}\right)^{\mathrm{T}} & =\frac{\hat{\Theta}^{\mathrm{m}}\left(\mathrm{X}_{1}^{0}+L, \mathrm{X}_{2}^{0}, \mathrm{t}\right)-\hat{\Theta}^{\mathrm{m}}\left(\mathrm{X}_{1}^{0}, \mathrm{X}_{2}^{0}, \mathrm{t}\right)}{K_{1} L} \\
& \simeq \frac{1}{2} \frac{K}{K_{1}}\left[\sum_{\mathrm{a}=\mathrm{I}}^{\mathrm{IV}}\left(\mathrm{G}^{\mathrm{a}} \mathrm{g}^{\mathrm{a}}+\mathrm{G}^{\mathrm{Ra}} \mathrm{g}^{\mathrm{Ra}}+\sum_{+,-}\left(\mathrm{G}_{ \pm}^{\mathrm{U}} \mathrm{g}_{ \pm}^{\mathrm{U}}+\mathrm{G}_{ \pm}^{\mathrm{D}} \mathrm{g}_{ \pm}^{\mathrm{D}}\right)\right]\right.
\end{aligned}
$$

where

$$
\begin{aligned}
& \mathrm{G}^{\mathrm{a}}=\left[\epsilon_{\theta}^{\mathrm{an}}-\frac{\mathrm{n}^{2}}{2}\left(Q_{31} \epsilon_{1}^{\mathrm{a}}+Q_{32} \epsilon_{2}^{\mathrm{a}}\right)\right] \\
& \mathrm{G}^{\mathrm{Ra}}=\left[\epsilon_{\theta}^{\mathrm{Ran}}-\frac{\mathrm{n}^{2}}{2}\left(Q_{31} \epsilon_{1}^{\mathrm{a}}+Q_{32} \epsilon_{2}^{\mathrm{a}}\right)\right] \\
& \mathrm{g}^{\mathrm{a}}=\hat{\mathrm{g}}^{\mathrm{a}}\left(\mathrm{X}_{2}^{0}, \mathrm{t}\right)=\frac{\sin \left(k_{1} L / 2\right)}{k^{K \mathrm{a}} L / 2} \cos \left(\frac{k_{1} L}{2}+k_{2}^{\mathrm{a}} \mathrm{X}_{2}^{0}-\omega \mathrm{t}+\varphi_{0}\right) \\
& \mathrm{g}^{\mathrm{Ra}}=\hat{\mathrm{g}}^{\mathrm{Ra}}\left(\mathrm{X}_{2}^{0}, \mathrm{t}\right)=\mathrm{g}^{\mathrm{a}}\left(\mathrm{X}_{2}^{0}, \mathrm{t}\right) \cdot \frac{k^{K \mathrm{a}}}{k^{K \mathrm{Ra}}} \\
& \mathrm{G}_{ \pm}^{\mathrm{U}}=\frac{1}{\mathrm{~T}_{ \pm}}\left[\sum_{\mathrm{a}=\mathrm{III}}^{\mathrm{IV}} \frac{-\mathrm{G}_{ \pm}^{\mathrm{a}} \mathrm{S}_{ \pm}^{\mathrm{a}}+\mathrm{G}_{ \pm}^{\mathrm{Rb}} \mathrm{S}_{ \pm}^{\mathrm{a} *}}{k^{K \mathrm{a}} L}+\sum_{\mathrm{a}=\mathrm{I}}^{\mathrm{II}} \frac{ \pm \mathrm{G}_{ \pm}^{\mathrm{a}} \mathrm{S}_{ \pm}^{\mathrm{a} *}+\mathrm{G}_{ \pm}^{\mathrm{Rb}} \mathrm{S}_{ \pm}^{\mathrm{a}}}{k^{K \mathrm{a}} L}\right]^{\frac{1}{2}} \\
& \mathrm{G}_{ \pm}^{\mathrm{D}}=\frac{1}{\mathrm{~T}_{ \pm}}\left[\sum_{\mathrm{a}=\mathrm{III}}^{\mathrm{IV}} \frac{\mathrm{G}_{ \pm}^{\mathrm{a}} \mathrm{S}_{ \pm}^{\mathrm{a} *}-\mathrm{G}_{ \pm}^{\mathrm{Rb}} \mathrm{S}_{ \pm}^{\mathrm{a}}}{k^{K \mathrm{a}} L}+\sum_{\mathrm{a}=\mathrm{I}}^{\mathrm{II}} \frac{\mp \mathrm{G}_{ \pm}^{\mathrm{a}} \mathrm{S}_{ \pm}^{\mathrm{a}}+\mathrm{G}_{ \pm}^{\mathrm{Rb}} \mathrm{S}_{ \pm}^{\mathrm{a} *}}{k^{K \mathrm{a}} L}\right]^{\frac{1}{2}} \\
& \mathrm{~g}_{ \pm}^{\mathrm{U}}=\hat{\mathrm{g}}_{ \pm}^{\mathrm{U}}\left(\mathrm{X}_{2}^{0}, \mathrm{t}\right)=\sin \left(\frac{k_{1} L}{2}\right) \cos \left[\frac{k_{1} L}{2} \mp\left(K_{2}-K_{2 \pm}^{\mathrm{h}}\right) \mathrm{X}_{2}^{0}-\omega \mathrm{t}+\varphi_{0} \pm\left(\Phi_{ \pm}^{\mathrm{U} 0}-\Phi_{0}\right)\right] \\
& \mathrm{g}_{ \pm}^{\mathrm{D}}=\hat{\mathrm{g}}_{ \pm}^{\mathrm{D}}\left(\mathrm{X}_{2}^{0}, \mathrm{t}\right)=\sin \left(\frac{k_{1} L}{2}\right) \cos \left[\frac{k_{1} L}{2} \pm\left(K_{2}-K_{2 \pm}^{\mathrm{h}}\right) \mathrm{X}_{2}^{0}-\omega \mathrm{t}+\varphi_{0} \pm\left(\Phi_{ \pm}^{\mathrm{D} 0}-\Phi_{0}\right)\right]
\end{aligned}
$$

Note that in the derivation of (85), the following approximation has been used

$$
\Theta^{\mathrm{m}}=\hat{\Theta}^{\mathrm{m}}=\left(\mathrm{X}_{1}, \mathrm{X}_{2}, \mathrm{t}\right) \simeq \frac{1}{2 E_{3}}\left(E_{3 \mathrm{~S}}+E_{3 \mathrm{~S}}^{\mathrm{R}}\right)
$$

\section{Interpretations of results}

The features of mode transition are shown in the Figs. 1 and 2. The wavenumber-frequency curves for several lowest guided TE mode are presented in Fig. 1. In this figure, superscripts o or e in the lable of each TE mode stand for either odd or even one, the number in subscript denotes the order of mode. Figure 2 represents the wavenumber-frequency relations for symmetric mode of guided elastic wave. For the mode transition from odd TE mode to another odd TE mode, the characteristic relation $\left(\mathrm{U}_{ \pm}=0\right)$ can be approximated as

$$
\frac{\left[\left(K_{1} \pm k_{1}\right)^{2}-\mu_{0} \varepsilon_{0} \Omega^{2}\right]^{\frac{1}{2}}}{\left[\mu_{0} \varepsilon_{33} \Omega^{2}-\left(K_{1} \pm k_{1}\right)^{2}\right]^{\frac{1}{2}}}=\tan \frac{\left[\mu_{0} \varepsilon_{33} \Omega^{2}-\left(K_{1} \pm k_{1}\right)^{2}\right] \mathrm{d}}{2}
$$




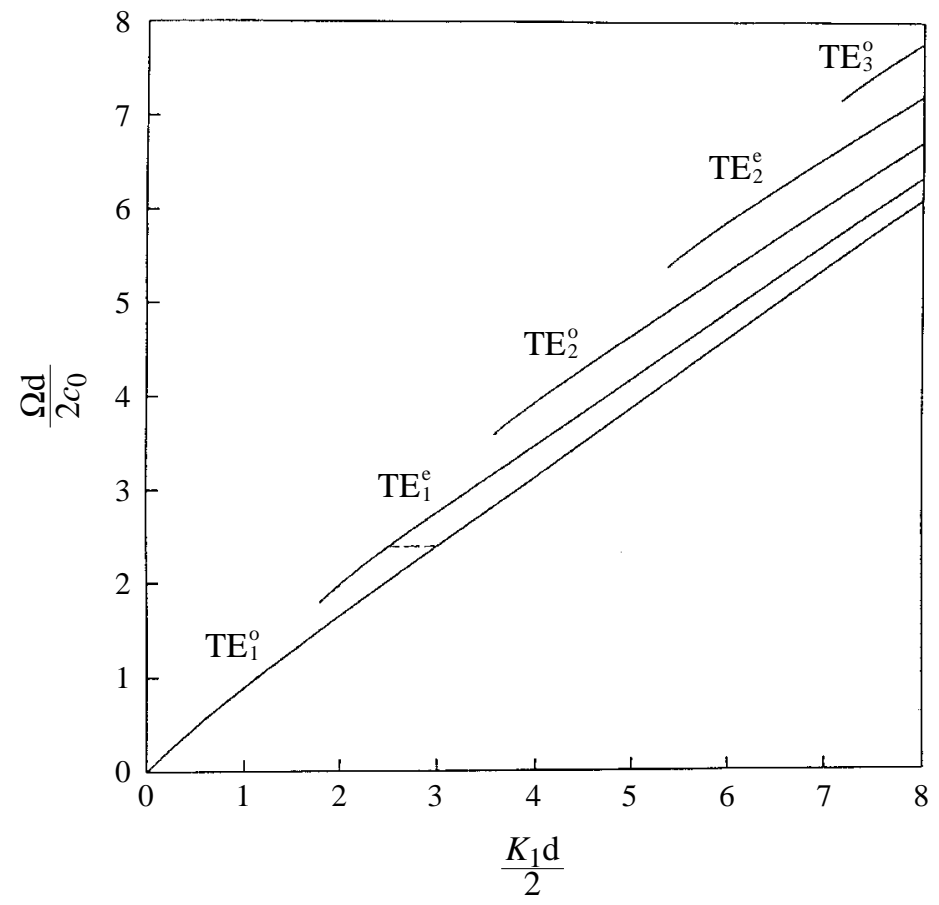

Fig. 1. The frequency spectrum of odd and even TE modes.

where the following estimation is used in the derivation of (88).

$$
\frac{\omega / \Omega}{k / K}=\frac{c_{e}}{c} \ll 1
$$

Therefore, the mode transition will occur under the condition:

$$
k_{1}=K_{1}^{(\mathrm{m} \pm 1)}-K_{1}^{(\mathrm{m})} \quad \mathrm{m}=1,2 \cdots
$$

where the superscript $\mathrm{m}$ stands for the order of mode. The equivalence in length for the marked lines in both Figs 1 and 2 will lead to the mode transition. For the both results discussed above, mode transition is dominated by elastic wave of slightly higher frequency $(k \sim \Delta K)$, and the phase shift is significantly governing by the elastic wave with lower frequency [15].

The mode transition for the cases that odd TE mode transfers to even TE mode or the mode of perturbed elastic wave is anti-symmetric can be analyzed by applying the similar procedure described in this paper.

\section{Conclusive remark}

In a dielectric slab waveguide, the guided electromagnetic wave which is disturbed by guided elastic waves has been investigated here. By the coupling between the particular solution and the homogeneous solution of the perturbed electromagnetic wave, the mode transition from the original guided electromagnetic mode to a new guided mode can be determined. Under the condition of no mode transition, the phase shift of original electromagnetic wave is calculated. The mode coupling of guided electromagnetic 


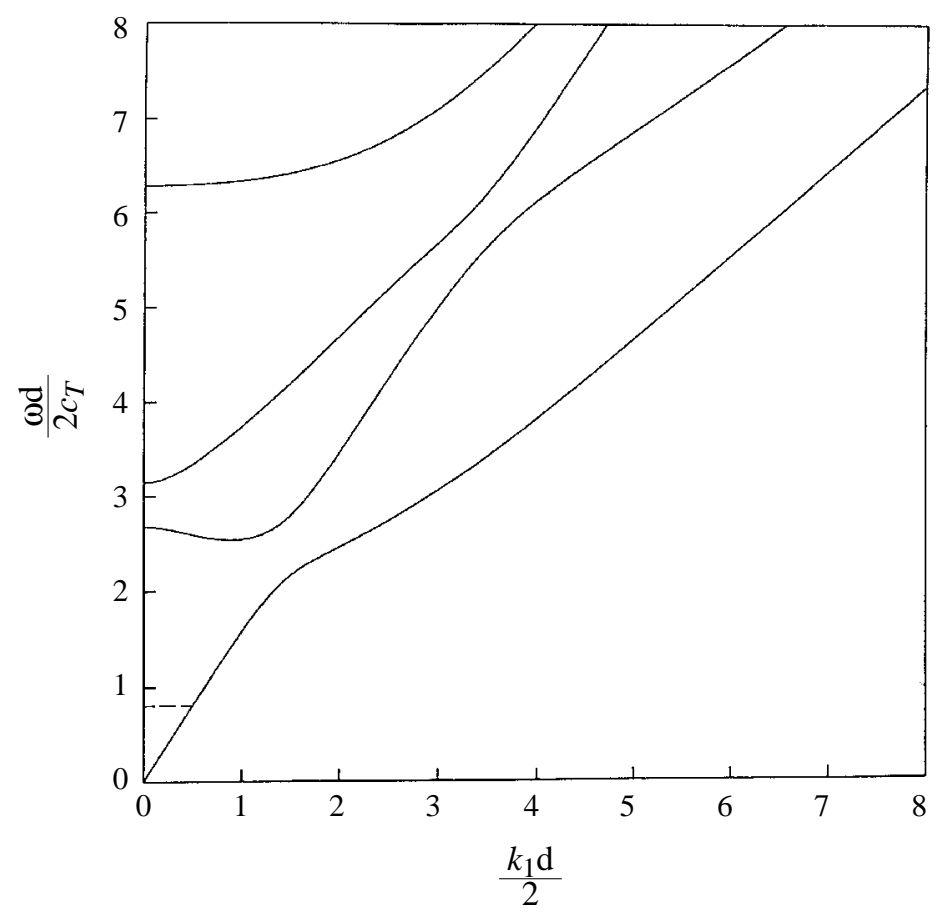

Fig. 2. The Rayleigh-Lamb frequency spectrum of symmetric modes.

modes in slab waveguide with slightly static deformation in surface has been investigated in Ref.17. Since the mechanical equation has not been involved, it is difficult to extend the couple mode theory in Ref.17 to dynamic disturbance.

The frequency shift in circular dielectric resonator [21] and the phase shift for various mode in fiber [22] have been discussed by the earlier researchers. Nevertheless, since the boundary conditions of the perturbed electromagnetic wave has not been counted, the mode transition have not been obtained by their approaches yet.

\section{References}

[1] J.A. Bucaro, N. Lagakos, J.H. Cole and T.G. Giallorenzi, in: Physical Acoustics, (Vol. XVI), W.P. Mason, ed., Academic Press, New York \& London, 1982, pp. 385.

[2] R. Hughes and J. Jarzynski, Appl. Opt. 19 (1980), 98.

[3] G.B. Hocker, Appl. Opt. 18 (1979), 1445.

[4] A.C. Eringen, in: Nonlinear Theory of Continuous Media, McGraw-Hill, New York \& San Francisco \& Toronto \& London, 1962.

[5] W.F. Brown, in: Magnetoelastic Interactions, Springer-Verlag, New York, 1966.

[6] Y.H. Pao and C.S. Yeh, Int. J. Engng. Sci. 11 (1973), 415.

[7] Y.H. Pao and K. Hutter, Proc. IEEE 63 (1975), 1011.

[8] K. Hutter and A.A.F van de Ven, in: Field Matter Interaction in Thermoelastic Solids, Springer-Verlag, Berlin, Heidelberg, 1978.

[9] G.A. Maugin, in: Continuum mechanics of electromagnetic solids, North-Holland, Amsterdam, 1988.

[10] A.C. Eringen and G.A. Maugin, in: Electrodynamics of Continua, Springer-Verlag, Berlin, Heidelberg, 1989.

[11] J.C. Baumhauer and H.F. Tiersten, J. Acoust. Soc. Am. 54 (1973), 1017.

[12] G.A. Maugin and J. Pouget, J. Acoust. Soc. Am. 68 (1980), 575.

[13] D.F. Nelson, in: Electric, Optic, and Acoustic Interaction in Dielectric, John Wiley \& Sons., 1979. 
76 C.-S. Yeh and C.-B. Lin / Mode transition of electromagnetic wave in slab waveguide subjected to in-plane elastic wave

[14] C.S. Yeh and C.B. Lin, Int. J. Appl. Electromagnetic Materials. 3 (1993), 269.

[15] C.S. Yeh and C.B. Lin, Int. J. Appl. Electromagnetic Materials. 5 (1994), 123.

[16] Y.H. Pao, in: Mechanics Today, (Vol. 4), S. Nemat-Nasser, ed., Pergamon Press, New York, 1978, pp. 209.

[17] D. Marcuse, in: Theory of Dielectric Optical Waveguides, Academic Press, New York \& London, 1974.

[18] D.K. Cheng, in: Field and wave Electromagnetics, Addison-Wesley P.C., 1983.

[19] R.F. Harrington, in: Time Harmonic Electromagnetic Fields, McGraw-Hill, New York, 1961.

[20] J.D. Achenbach, in: Wave Propagation in Elastic Solids, North-Holland P.C., Amsterdam \& London, 1973.

[21] P.C.Y. Lee, J.S. Wang and A. Ballato, Mechanics of Electromagnetic Materials and Structure8s, AMD-161/MD-42 (1993), 129.

[22] G.B. Hocker and W.K. Burns, IEEE J. Quantum Electron QE-11 (1975), 270. 